\title{
Two-Dimensional Borophene: Properties, Fabrication, and Promising Applications
}

\author{
Zhongjian Xie, ${ }^{1,2}$ Xiangying Meng, ${ }^{2}$ Xiangnan $\mathrm{Li}^{3}$ Weiyuan Liang, ${ }^{1}$ Weichun Huang $\mathbb{D},{ }^{4}$ \\ Keqiang Chen, ${ }^{1}$ Jianming Chen, ${ }^{1}$ Chenyang Xing $\mathbb{D},{ }^{5}$ Meng Qiu, ${ }^{6}$ Bin Zhang, ${ }^{1}$ Guohui Nie, \\ Ni Xie, ${ }^{1}$ Xiaobing Yan, ${ }^{3}$ and Han Zhang $\mathbb{D}^{1}$ \\ ${ }^{1}$ Key Laboratory of Optoelectronic Devices and Systems of Ministry of Education and Guangdong Province, Institute of Microscale \\ Optoelectronics and Otolaryngology Department and Biobank of the First Affiliated Hospital, Shenzhen Second People's Hospital, \\ Health Science Center, Shenzhen University, Shenzhen 518060, China \\ ${ }^{2}$ Shenzhen International Institute for Biomedical Research, 518116 Shenzhen, Guangdong, China \\ ${ }^{3}$ National-Local Joint Engineering Laboratory of New Energy Photovoltaic Devices, Key Laboratory of Digital Medical Engineering of \\ Hebei Province, College of Electron and Information Engineering, Hebei University, Baoding 071002, China \\ ${ }^{4}$ Nantong Key Lab of Intelligent and New Energy Materials, College of Chemistry and Chemical Engineering, Nantong University, \\ Nantong, 226019 Jiangsu, China \\ ${ }^{5}$ Center for Stretchable Electronics and Nanoscale Systems, Key Laboratory of Optoelectronic Devices and Systems of Ministry \\ of Education, College of Physics and Optoelectronic Engineering, Shenzhen University, Shenzhen 518060, China \\ ${ }^{6}$ Key Laboratory of Marine Chemistry Theory and Technology (Ocean University of China), Ministry of Education, \\ Qingdao 266100, China
}

Correspondence should be addressed to Ni Xie; xn100@szu.edu.cn, Xiaobing Yan; yanxiaobing@ime.ac.cn, and Han Zhang; hzhang@szu.edu.cn

Received 13 April 2020; Accepted 11 May 2020; Published 15 June 2020

Copyright (c) 2020 Zhongiian Xie et al. Exclusive Licensee Science and Technology Review Publishing House. Distributed under a Creative Commons Attribution License (CC BY 4.0).

\begin{abstract}
Monoelemental two-dimensional (2D) materials (Xenes) aroused a tremendous attention in 2D science owing to their unique properties and extensive applications. Borophene, one emerging and typical Xene, has been regarded as a promising agent for energy, sensor, and biomedical applications. However, the production of borophene is still a challenge because bulk boron has rather intricate spatial structures and multiple chemical properties. In this review, we describe its excellent properties including the optical, electronic, metallic, semiconducting, photoacoustic, and photothermal properties. The fabrication methods of borophene are also presented including the bottom-up fabrication and the top-down fabrication. In the end, the challenges of borophene in the latest applications are presented and perspectives are discussed.
\end{abstract}

\section{Introduction}

Graphene is the first discovered 2D material [1]. The discovery of the astonishing properties of graphene has brought forth a series of new materials known as " $2 \mathrm{D}$ materials" [2-4]. 2D forms are a comparatively exciting and new area for many applications $[5,6]$. Usually, 2D materials have many prominent physical properties that are promising for electronic devices, nanoengineering, energy conversion, and photonics [7-11]. With the rapid development of graphene, 2D materials, such as phosphorene, BN, germanene, antimonene, silicene, arsenene, and transition metal dichalcogen- ides, have recently arisen extensive interest. A mass of atom-thick materials have been theoretically predicted or synthesized [12-16]. More surprisingly, they possess different structures from graphene, owing to the different outof-plane buckling degrees [17]. Besides the 2D materials exfoliated from their layered bulk counterparts, some 2D materials can also be manufactured from the bulk materials without layered form, such as the compound of $2 \mathrm{D}$ flat boron, 2D GaN, and hafenene [18, 19].

The research on boron in various compounds can be traced back to several hundred years ago, because boron possesses the extraordinary property to combine with nearly 
all of the other elements. Among them, hexagonal boron nitride (h-BN) is a wide bandgap III-V compound. It is a layered material with a graphite-like structure in which planar networks of h-BN hexagons are regularly stacked. h-BN possesses a high chemical stability, excellent physical properties, and a high thermal conductivity [20-23]. It is very similar to graphite, so that one may expect to prepare pure boron. In Sands' work, the pure boron was first documented in 1957 and the bulk g-B106 with an extremely complicated structure was reported. Up to now, bulk boron is widely known to have more than 16 polymorphs, all featuring interlinked polyhedra but only a few having identified crystal structure [17].

Since boron locates between nonmetallic carbon and metallic beryllium, there are merely three valence electrons in boron: $[\mathrm{He}] 2 \mathrm{~s}^{2} 2 \mathrm{p}^{1}$. The $2 \mathrm{p}$ electron and its orbit radius are near the $2 \mathrm{~s}$ state, endowing it both metallicity and nonmetallicity. In bulk boron, the particular electronic structure empowers the formation of greatly diverse bonding and facilitates the extraordinary bond formation. 2D boron provides greater energy relief, compared to any other $2 \mathrm{D}$ materials $[17,24]$.

In 2015, the 2D boron sheet was successfully fabricated on argentum (Ag) substrates [25]. The study of borophene has attracted a lot of researchers in many fields, such as material science, nanotechnology, physics, chemistry, and condensed matter $[13,26,27]$. "Borophene" is the potential new atom-thick boron nanosheets for the large-scale synthesis [28]. It is the lightest 2D material to date. Borophene is the neighbor of graphene, and thus, it is desired to possess some similar properties to graphene [29]. Both $\sigma$ and $\pi$ electrons in borophene occupy the electronic states of the Fermi surface, making it superconductive. There are no high pressure and external strain; borophene could have the highest $T_{c}$ among the $2 \mathrm{D}$ materials. For $2 \mathrm{D}$ boron structures, the chemical and structural complexity, electronic properties, and stability have been studied extensively [27, 30, 31].

The mechanical properties of borophene are particularly interesting and important. Firstly, borophene has low mass density. Provided that its ideal strength and in-plane stiffness are satisfactorily high, borophene can be used as assist elements for designing composites. Secondly, borophene is suitable for fabricating flexible nanodevices because of the high standards of flexibility against off-plane deformation [32-34]. Moreover, because of the powerfully anisotropic structure in borophene, its magnetic and electronic properties can be effectively controlled for multiple applications [35-37]. As the boron atoms are rich in bonding configurations, borophene is polymorphic, further differentiating it from other 2D materials $[38,39]$. The low mass density of boron also results in the strong electron-phonon coupling, within the scope of $10-20 \mathrm{~K}$ which causes phonon-mediated superconductivity with high critical temperature $[40,41]$. In a word, borophene is rich in resources, has low atomic weight, is lightweight, is low cost, and has excellent electrical performances. These advantages of borophene provide it more possibilities for practical application in the future.

Although borophene has many potential applications, the synthesis and discovery of its neoatomic structures with well-designed structure-property relationships retain among

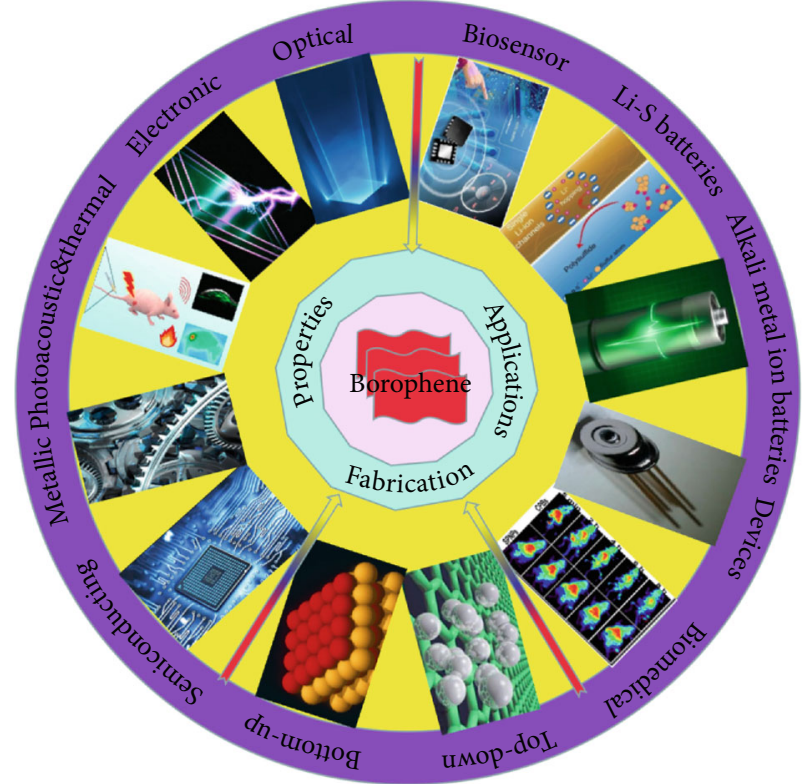

Figure 1: Comprehensive overview diagram of borophene.

the most severe challenges. In extra, for synthetic $2 \mathrm{D}$ materials, the resulting atomic structure is influenced by multiple factors, such as the constituent elements, processing conditions, and growth substrates [7]. In order to realize practical applications, insured synthesis of quality specimens and the separation of borophenes from substrates remain challenging, requiring continuing experimental and theoretical efforts [17].

In this review, we introduced the different experimental fabrication methods, the physicochemical properties, and the latest applications of borophene (Figure 1). The experimental synthesis includes bottom-up fabrication and top-down fabrication. The physicochemical properties of borophene mainly contain the optical, electronic, semiconducting, photoacoustic and photothermal, and metallic properties. Finally, we summarized the application of borophene in many fields, such as Li-S batteries, alkali metal ion batteries, and sensor and biomedical applications.

\section{Theory and Properties}

2.1. Optical Properties. The complex dielectric function of borophene benzene is $\varepsilon(\omega)=\varepsilon_{1}(\omega)+i \varepsilon_{2}(\omega)$, which determines its optical properties. In the case of metals, the sum of interband and intraband components constitutes the dielectric tensor. We do not take into account factors other than visible regions and interband transitions, so the dielectric function in druid region (low frequency) (1), (2) may not be accurate. The imaginary part $\varepsilon_{1}^{\alpha \beta}(\omega)=1+(2 / \pi) P \int_{0}^{\infty}$ $\left(\varepsilon_{2}^{\alpha \beta}\left(\omega^{\prime}\right) \omega^{\prime} / \omega^{\prime 2}-\omega^{2}+i \eta\right) d \omega^{\prime},(2)$ of the mediation tensor is confirmed by summing the empty band parts using (3).

$$
\varepsilon_{2}^{\alpha \beta}(\omega)=\frac{2 \pi e^{2}}{\Omega \varepsilon_{0}} \sum_{k, v, c} \delta\left(E_{k}^{c}-E_{k}^{v}-h \omega\right)\left|\left\langle\Psi_{k}^{c}|u \cdot r| \Psi_{k}^{v}\right\rangle\right|^{2}
$$



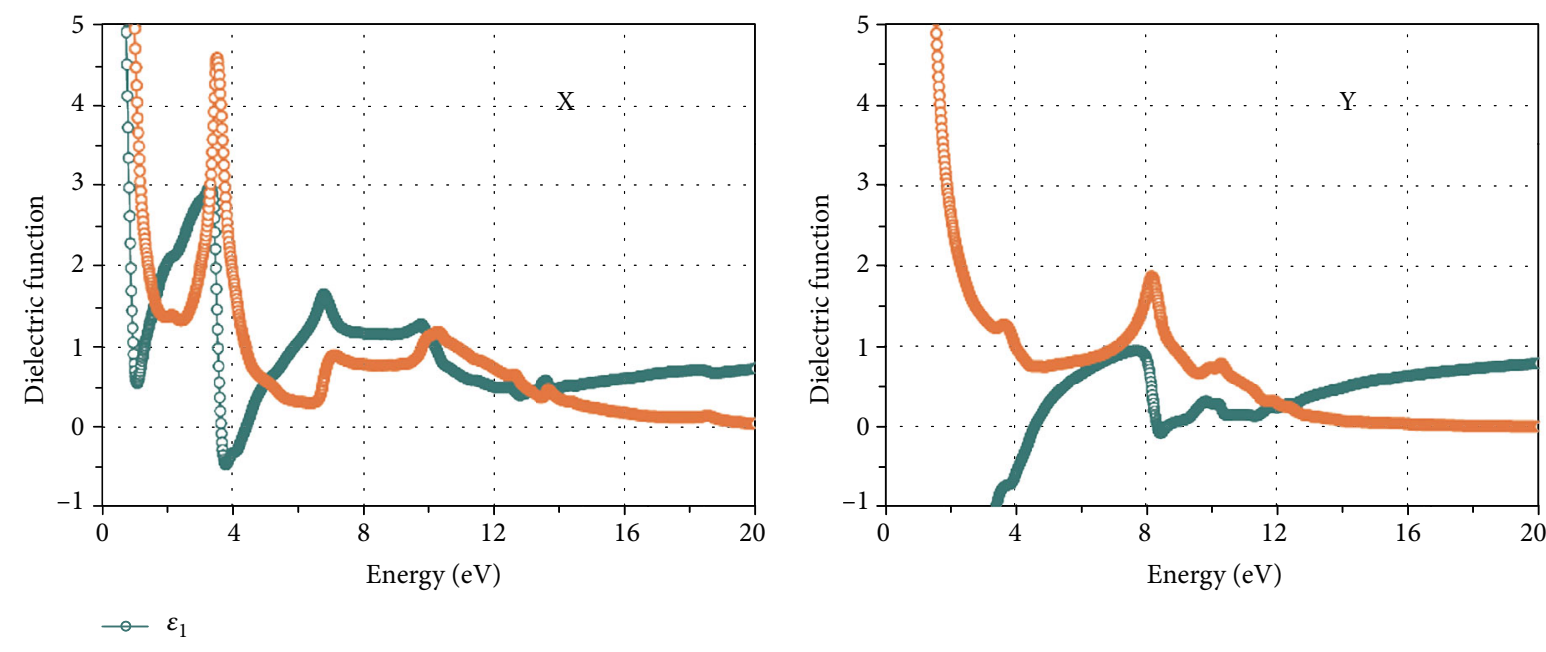

(a)
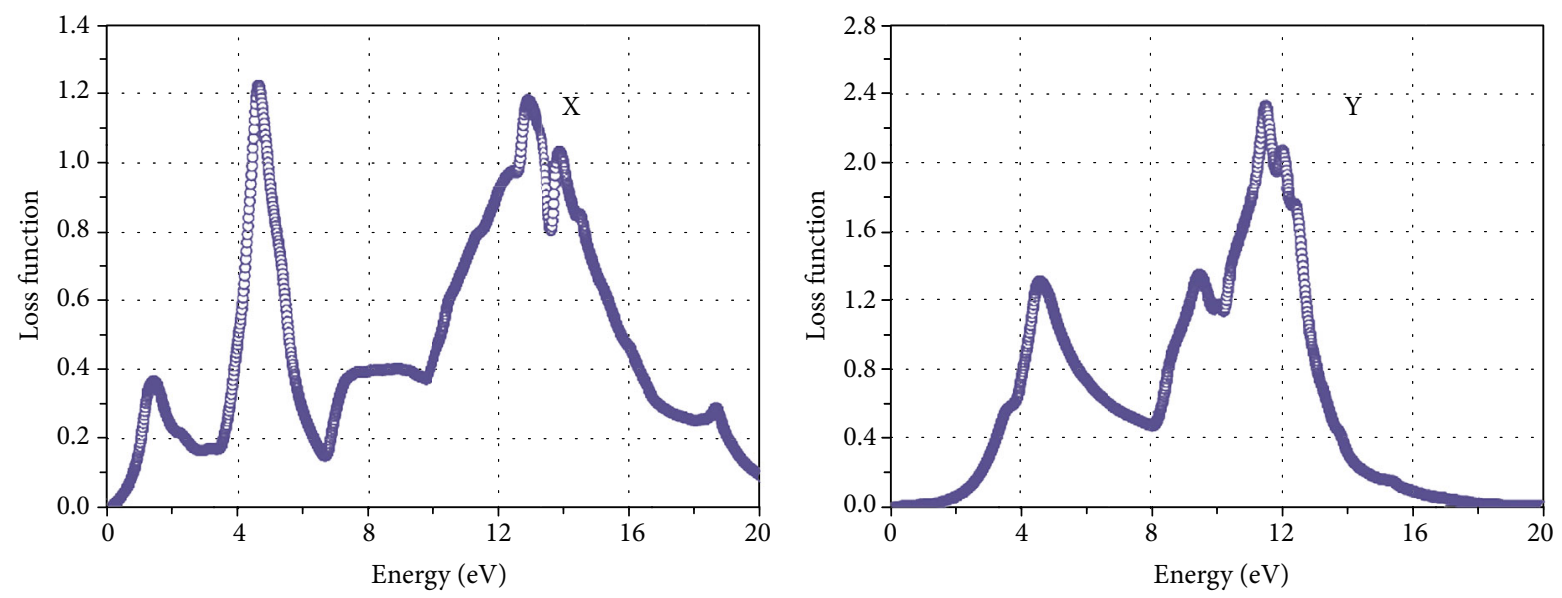

(b)

Figure 2: The optical response of polarized light on the $X$ and $Y$ axes to the illumination of 2D borophene cells, as illustrated in (a) and (b). Reprinted with permission from Ref. [35]. Copyright 2016 Royal Society of Chemistry.

$\varepsilon_{0}$ is the dielectric constant of the vacuum, $\omega$ is for volume, $v$ and $c$ explicit the $E_{v}$ and $E_{c}$, $\omega$ express the energy of the object phonon, $u \cdot r$ stands for momentum symbol, and $\Psi_{k}^{c}$ and $\Psi_{k}^{v}$ express the conduction band and valence band at point $K$. The real part $\varepsilon_{1}^{\alpha \beta}(\omega)$ of the dielectric tensor is obtained from the Kramers-Cronin relation:

$$
\varepsilon_{1}^{\alpha \beta}(\omega)=1+\frac{2}{\pi} P \int_{0}^{\infty} \frac{\varepsilon_{2}^{\alpha \beta}\left(\omega^{\prime}\right) \omega^{\prime}}{\omega^{\prime 2}-\omega^{2}+i \eta} d \omega^{\prime} .
$$

The absorption constant $\alpha(\omega)$ and $R(\omega)$ can be calculated from functions (4) and (5) according to the above-mentioned optical characteristics of borophene such as dielectric function and energy loss spectrum $L(\omega)$.

$$
L(\omega)=\operatorname{Im}\left(-\frac{1}{\varepsilon(\omega)}\right)=\frac{\varepsilon_{2}(\omega)}{\varepsilon_{1}^{2}(\omega)+\varepsilon_{2}^{2}(\omega)}
$$

$$
\begin{aligned}
& \alpha(\omega)=\frac{\sqrt{2} \omega}{c}\left\{\left[\varepsilon_{1}^{2}(\omega)+\varepsilon_{2}^{2}(\omega)\right]^{1 / 2}-\varepsilon_{1}(\omega)\right\}^{1 / 2}, \\
& R(\omega)=\left|\frac{\sqrt{\varepsilon_{1}(\omega)+i \varepsilon_{2}(\omega)}-1}{\sqrt{\varepsilon_{1}(\omega)+i \varepsilon(\omega)}+1}\right|^{2} .
\end{aligned}
$$

By calculating the dielectric equation and the electron energy loss equation of the incident radiation after the polarity of the electric field vector $E$ in the directions of $a$ and $b$, it can be seen that the crystal structure of borophene is anisotropic, resulting in the anisotropy in its optical properties.

In Figure 2(a), the virtual section of the dielectric medium model function quickly decreases at small frequencies close to the area of free electron. Direction of light polarization $X, \varepsilon_{2}(\omega)$, increases fast at $2.41 \mathrm{eV}$ and then up to the peak at $3.52 \mathrm{eV}$. Interband transitions from many occupied $K$ states under electromotive force appear at the consistent energy, resulting in a powerful spike for direction of light 

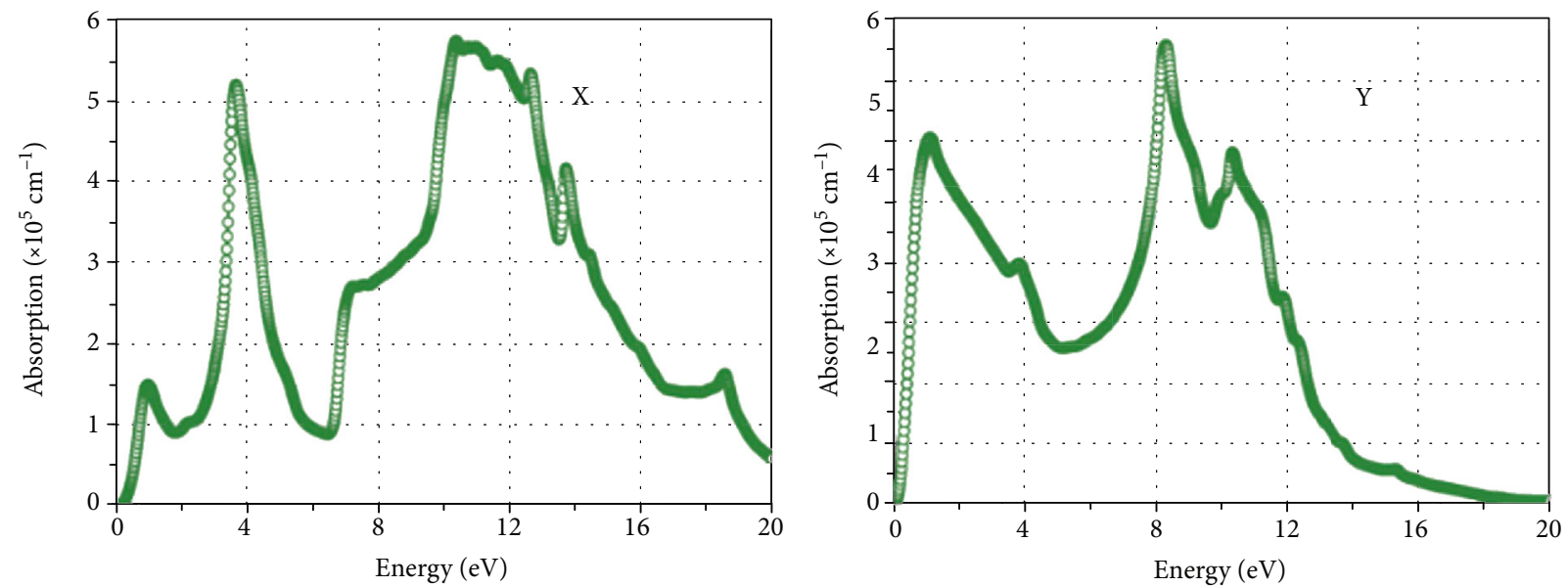

(a)
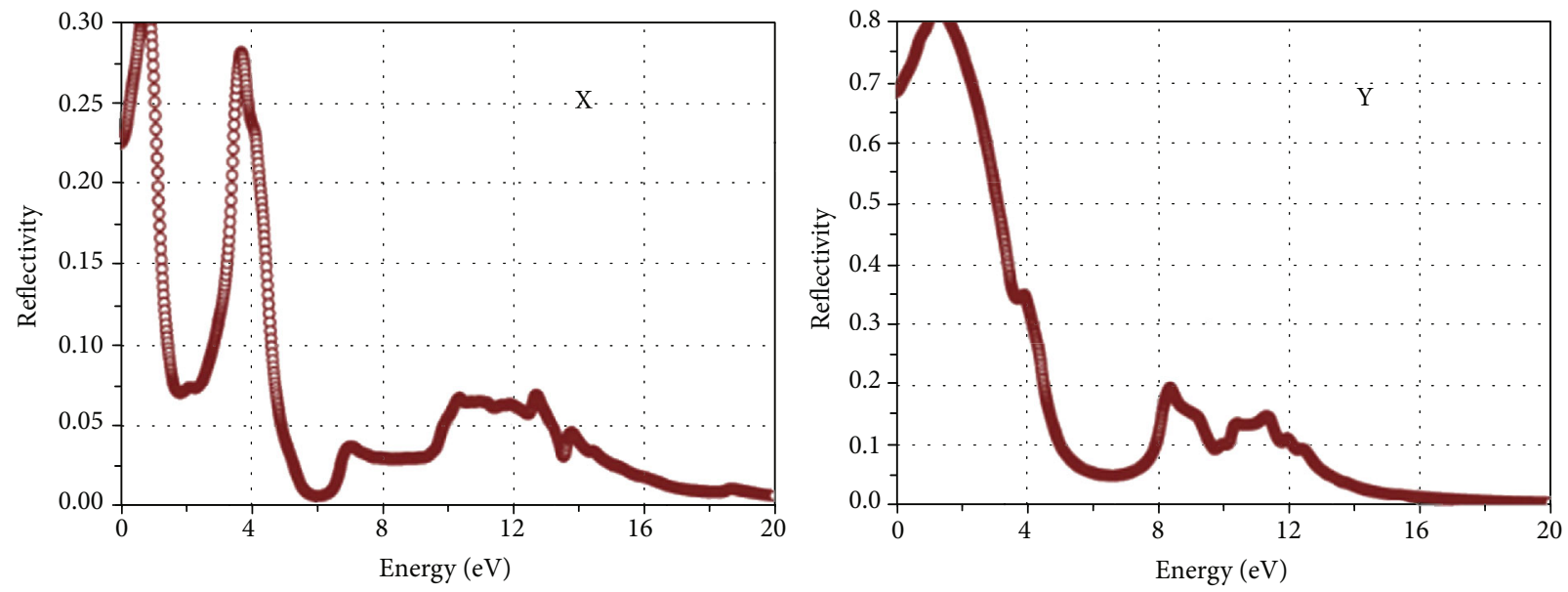

(b)

FIGURE 3: (a) Absorption coefficient of borophene and (b) reflectivity alongside the different two directions. Reprinted with permission from Ref. [35]. Copyright 2016 Royal Society of Chemistry.

polarization $Y$ and the peak $\varepsilon_{2}(\omega)$ is stronger than other peaks at $8.14 \mathrm{eV}$.

Due to the anisotropic crystal structure of borophene, there is a huge in-plane anisotropy discovered in the optical properties [13]. Borophene is a material showing promising potential in photovoltaic, flexible electronics, and display technologies, on account of the high electrical conductivity and optical transparency along the a direction, and all kinds of novel anisotropy [35, 42, 43].

Tai et al. have measured the optical bandgap of boron, which was $2.25 \mathrm{eV}$. It is approaching the value affirmed by first-principles calculations, which was $2.07 \mathrm{eV}$. They observed through strong photoluminescence and found that the borophene is a charming direct bandgap semiconductor [44]. Their ability to emit and absorb light of semiconductors is directly influenced by the electronic band structures. Wang et al. found that, the direct bandgap semiconductors, photons with energy surpass the bandgap energy which could be easily emitted or absorbed. For indirect bandgaps, there must be an additional phonon emitted or absorbed to make up for the energy imbalance, making the efficiency of photon emission or absorption process decreased [5].
Figure 3 reveals the absorption coefficient and reflectivity. In the two plane directions, the main absorptions are in the different positions. Along a direction, the major absorption peaks are situated at about 10.36 and $3.65 \mathrm{eV}$. Along $\mathrm{b}$ direction, the absorption regions can be up to $10.31,8.29$, and $1.09 \mathrm{eV}$. Moreover, the reflectivity of borophene along the two directions is lesser than $30 \%$ and higher than $40 \%$, respectively, in the visible region. Along the uncorrugated a direction, the transmittance of borophene is high, and the electrical conductivity is very high. However, in the visible area, the optical conductivity of borophene is very small. Such properties offer chances for applications in flexible electronics, photovoltaic, and display technologies [35].

Adamska et al. find that borophene is an anisotropic metal, with a faint absorbance in the visible area, thicknessdependent optical transparency, and strong energy [45]. Tatullo et al. demonstrate that in the visible range, borophene is a weak absorber, which holds high optical transparency, resulting in excellent transparency [46]. Moreover, Lherbier et al. show a strong photosensitivity of borophene as regards any surface modifications [47]. 


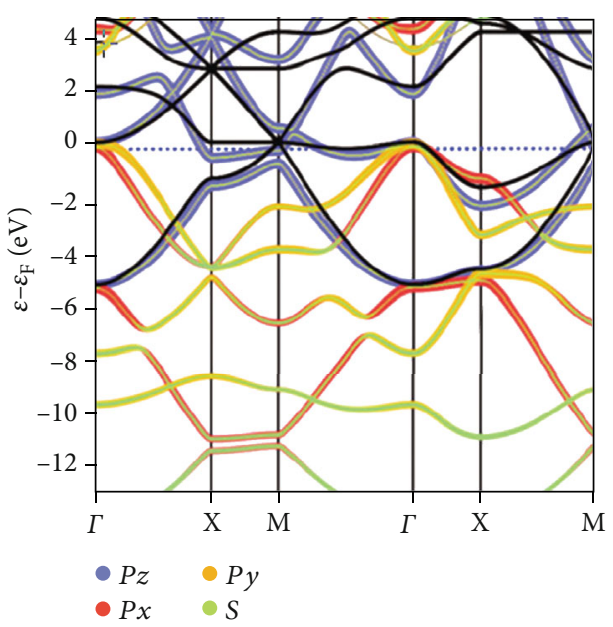

(a)

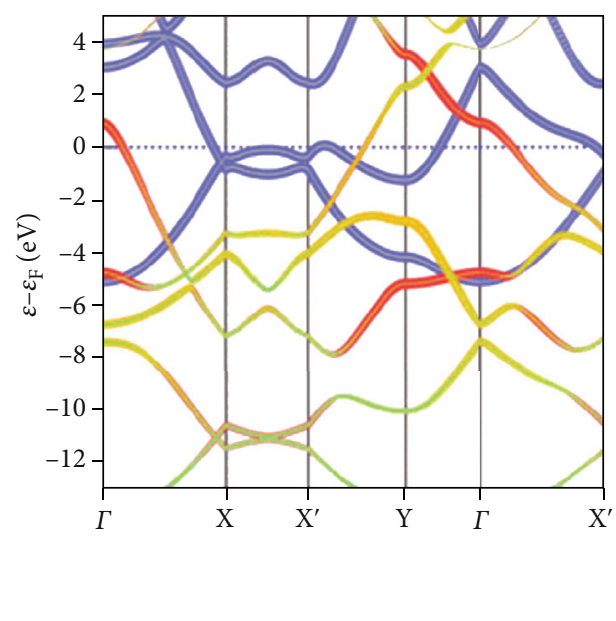

(b)

FIGURE 4: Electronic properties of 2D boron. The local density approximation band structures of the (a) $v 1 / 6$ and (b) $v 1 / 5$ sheets in a vacuum. Reprinted with permission from Ref. [49]. Copyright 2016 American Chemical Society.

2.2. Electronic Properties. The crystal structure of borophene is highly anisotropic [35]. Lots of researches foretell that different low-energy crystal structures may lead to metallic or semimetallic behavior. They may include Dirac cones near or at the Fermi energy and show anisotropy of conductivity because of their anisotropic bonding configuration $[45,48]$. 2D boron exhibits diversiform structural polymorphs. Although the 2D and 3D forms of boron are alike in the organizations of chemical bonds, every $2 \mathrm{D}$ boron polymorph is metallic but the nature of their 3D forms is diverse [17].

The band structures of the $v 1 / 6$ and $v 1 / 5$ 2D boron sheets computed employing the LDA are shown in Figures 4(a) and $4(\mathrm{~b})$. Most of the states are metallic root in the $2 \mathrm{pz}$ state, around the Fermi level, which is greatly delocalized above a huge energy window. Under circumstance of the $v 1 / 52 \mathrm{D}$ boron sheet, the metallicity is given by the $2 \mathrm{px}$ and 2 py states, which will generate a bandgap close to the Fermi level in the $2 \mathrm{D}$ boron sheets with the value of $v$ between $10 \%$ and $15 \%$. The estimated metallicity was confirmed by the latest experimental methods [17].

Figure 5(a) presents the band structure of borophene calculated with a settled Fermi level (EF), by the PBE functional in the direction of high symmetry of Brillion area, which is keeping with former theoretical work. The EF consists of three different bands, two follow the G-X direction and the other one follows the S-Y direction, along the directions indicating the metallic behaviors [35]. The crystal structure and the electronic band structure all reveal high anisotropy. The band structure displays metallic character while along one direction; nevertheless, a huge one is found while along the other direction [13]. Therefore, layered borophene behaves anisotropically in electronic properties rooting the anisotropic atomic structure [50].

It is important to obtain the exact electronic structure of borophene by hybrid functional calculations employing the HSE06 functional calculation. The band structure of borophene is shown in Figure 5(b). Compared with PBE functional calculation, the bandgap in HSE06 functional calculation increased by more than $11 \mathrm{eV}$, which was caused by the slight growth of conduction band minimum value (CBM) and the slight reduction of valence band maximum value (VBM) along the X-S direction [35].

Figures 5(c) and 5(d) show the optical absorption spectrum obtained by the imagined part of the dielectric function. It is found that two plasma branches exist in different energy ranges, that is, the high-energy branch (HE mode) and low-energy branch (LE mode), respectively. The HE mode is approximately linearly diffused and can extend to the ultraviolet region. However, the LE mode branch presents a relatively obvious anisotropic dispersion in different directions [51].

Because of the electron deficiency of elemental boron, it has all kinds of crystal structures including multicenter bonds. In contrast with the metallic, ionic, covalent, and van der Waals bonds, the multicenter bonds have extremely complex bonding type, which is vital because of its existence in abundant compounds [53]. The line defects in borophene have similar metallic structure to the original $v 1 / 6$ and $v 1 / 5$ sheets; thus, the electronic properties of borophene are relatively stable at room temperature for its underlying structural complexity. However, the delicate electronic modulations consistent with a CDW are prominent in the extremely low temperature $[54,55]$. The chemical and electronic properties of borophenes are possible to be tuned by various chemical modifications. Consequently, the novel borophene and graphene may be the complementary partner [28].

2.3. Photoacoustic and Photothermal Properties. Usually, photoacoustic and photothermal signals are used for imagingguided therapy [56-58]. In the very attractive area of nanomaterial-based cancer therapy, nanomaterials with distinct properties, for instance photothermal and photoacoustic transfer principles, have been deemed as an amusing and hopeful approach for the destruction of cancer cells $[59,60]$.

Kang et al. have announced the photoacoustic effect of small-sized material in an aqueous solution and studied the 


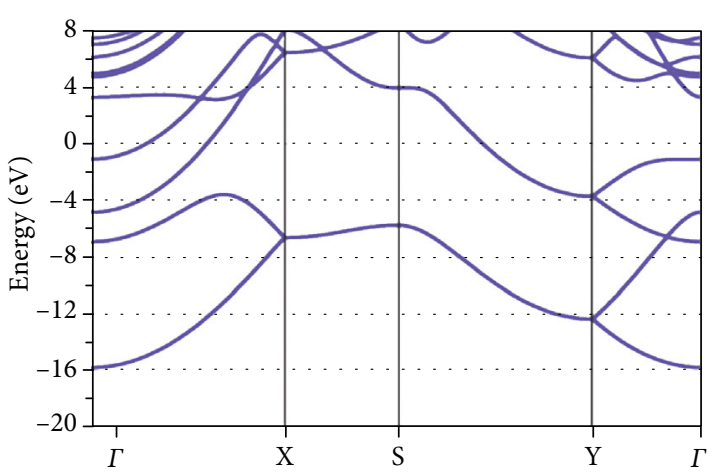

(a)

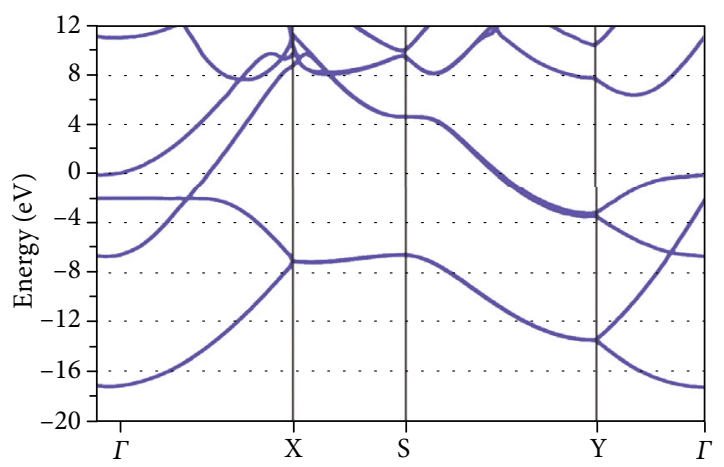

(b)

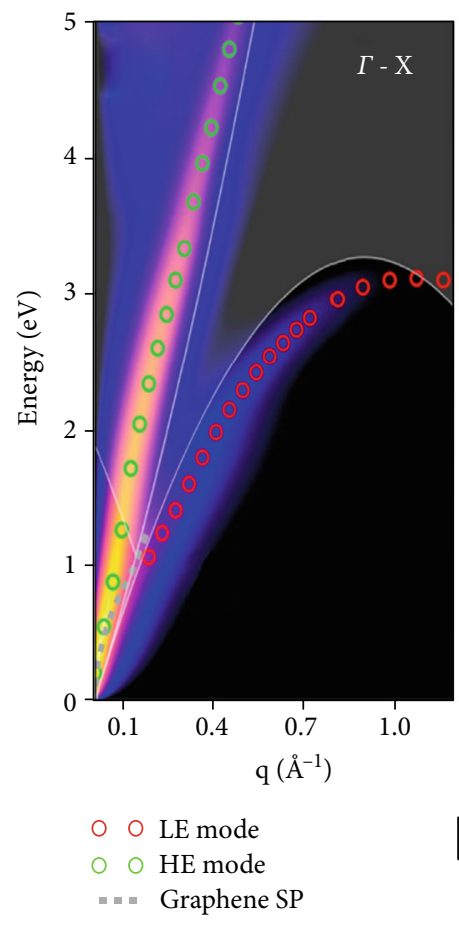

(c)

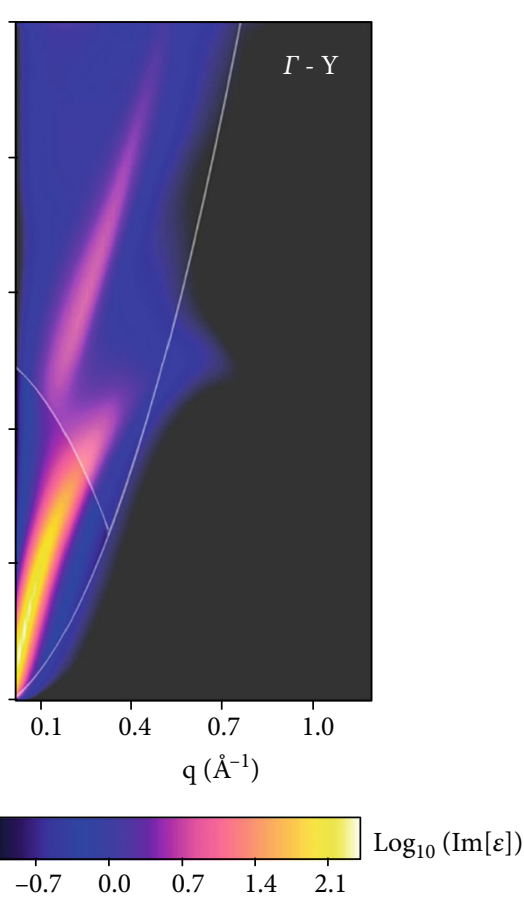

(d)

FIGURE 5: The electron band structure of borophene through (a) PBE and (b) HSE06 functional, respectively. Reprinted with permission from Ref. [35]. Copyright 2016 Royal Society of Chemistry. (c, d) The I-X and Г-Y directions of dielectric function. Reprinted with permission from Ref. [52]. Copyright 2009 American Physical Society.

mechanism [61]. The intensity of photoacoustic amplitude produced a forceful shock wave and led to explosion like a firecracker at the nanoscale. The conversion from optical energy to acoustic energy could lead to a new discovery for using small-sized material as underlying therapeutic agents for cancer cell destruction [59].

For example, borophene can enter the cell at a very small size; the photoacoustic energy can be used both for cancer therapeutics and to generate acoustic waves on smallgrained materials; it can lay a foundation for the application of efficient optical image generation in the future. The stress and pressure coming into being on the surface of nanomaterial during the photoacoustic therapy process can also be applied to photocontrolled release of anticancer drugs, iRNA, and proteins from the surface of small-sized material into the cells. Cancer is killed without giving rise to drug resistance and toxicity since such a photoacoustic process is a physical response that occurs in a short period of time. These new discoveries will be useful for the application of the photoacoustic properties and small-sized material structures in cancer therapeutic approaches [59].

The in vivo and in vitro results presented by Ji et al. proved the huge potential of the B-PEG (boron surface modification with polyethylene glycol) nanosheet for cancer photothermal chemotherapy [62]. They also developed the potential of the near-infrared light-induced hyperthermia of
B-PEG nanosheet. The temperature of aqueous solution contains boron which was much higher than the pure aqueous solution under the same condition of NIR laser. The huge temperature variation further confirmed that our efforts are needed for preparation of the single-layer borophene. Because of its high photothermal conversion efficiency, boron nanosheet can be developed into effective materials for tumor treatment [62].

2.4. Metallic Properties. Metallicity is the most famous character of borophene in comparison with other semiconductors (e.g., phosphorene) or semimetals (e.g., silicene and graphene). Differing from bulk boron allotropes, borophene reveals metallicity which is in consistence with the anticipations of a greatly anisotropic 2D metal [25].

STS notarizes the metallicity of borophene through current-voltage curves, as shown in Figure 6(a), and the dI/ $d V$ spectra, as shown in Figure 6(b), which measure the DOS [25]. On the one hand, borophene is able to resist a large load, until the failure. On the other hand, the reactivity of borophene helps covalent bonding to the base that capacitates useful load transfer. Plenty of structural information on borophene also promoted researches on their electronic transport capacities. Meanwhile, if it holds a 2D structure, boron starts to display interesting metallic properties [46]. In particular, borophene could take along a high conductive 


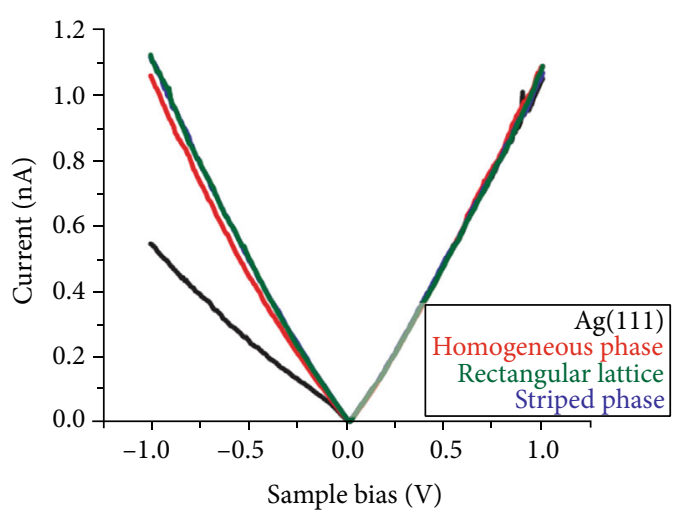

(a)

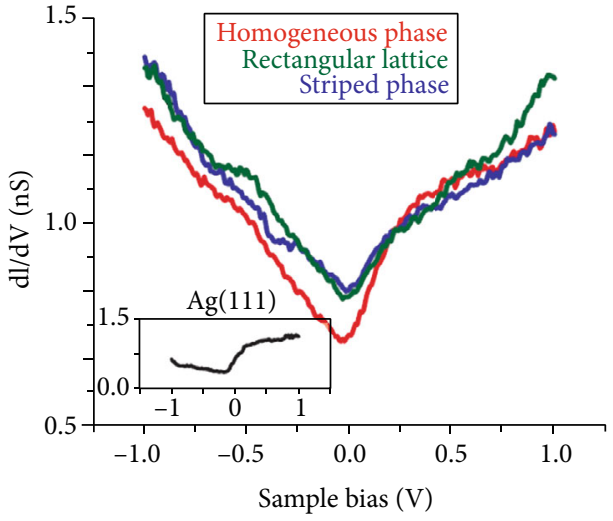

(b)

FiguRE 6: (a) The scanning tunneling spectroscopy (STS) current-voltage curves of borophene and (b) the scanning tunneling spectroscopy $d I / d V$ spectra of borophene. Reprinted with permission from Ref. [25]. Copyright 2015 American Association for the Advancement of Science.

electron density, $\sigma$, which does not depend on the Fermi level, in the near-visible range, opening a door to extend the plasmon energy [17].

The lattice parameters of borophene are shown in Figures $7(\mathrm{a})-7(\mathrm{c})$, with the value of $a=1.6212 \AA$ and $b=$ $2.8699 \AA$. The value difference between bottom and top atoms can be up to $0.89 \AA$. Fermi velocity plays an important role in electrical conductivity, the electron-phonon relaxation time close to EF, and DOS at EF [47]. Figure 7(d) shows the electronic band structure, which reveals a priori and powerful anisotropic metallic feature as the bands are discovered to be deeply scattered in the $k_{x}$ direction ( $\Gamma-\mathrm{X}$ and $\mathrm{Y}-\mathrm{S}$ ), accompanied large group velocity at the value of $6.6 * 10^{5}$ m pers. Figure $7(\mathrm{e})$ reveals that electronic bands can often pass the Fermi level if a line is parallel to $k_{x}$; however, it is different to the lines that were parallel to $k_{y}$. Thus, the prediction was that transport may only happen till the choosy wave vectors $k$ whose $k_{x}$ constituent was in the permitted region. In the end, the recalculation and correction of the band structure were conducted by using the LDA method with ABI-NIT package and a one shot $\mathrm{G}_{0} \mathrm{~W}_{0}$ approximation to the quasi particle problem. Kohn-Sham structure showed less different change to the $\mathrm{G}_{0} \mathrm{~W}_{0}$ one. One can only realize an increase of the local bandgap at the $\Gamma$ point and usually speaking an increase of the Fermi velocity $[35,47]$.

Mir et al. guess that due to the slight weight of boron, it may solidify electron-phonon combination; this may improve conventional, phonon-mediated superconductivity [17]. Boron reveals much more crossings through deeply dispersed (almost parabolic) bands. It indicates that compared to metallic $\mathrm{MX}_{2}$ compounds, $2 \mathrm{D}$ boron owns a huge free charge shipper concentration [63]. Therefore, borophene may be very useful as an electrode material in the future [54]. Moreover, after oxidation, borophene remains metallic and the oxidized borophene has an enormous improvement on both optical conductivity and optical property [47].

2.5. Semiconducting Properties. In recent years, due to the rapid development of the electronic industry, the traditional silicon technology can no longer meet the requirements of the semiconductors [64]. Hence, looking for a new semiconductor material to promote the development of the electronic industry is a crucial factor. Through research, Yang et al. found that borophene can form ideal contact with 2D semiconductor and effectively reduce the contact resistance, which can further improve the related performance of $2 \mathrm{D}$ transistor [34].

Jie et al. studied experimentally and systematically that the tunnel barrier was nearly zero after borophene making effective contact with various $2 \mathrm{D}$ semiconductors. It is shown in Figures 8(a)-8(d). The valid channel barrier height is given as the barrier height difference that must be overcome when the Fermi energy of the carrier in the metal is the same as that of the heterostructure. As shown in Figure 8(e), all of the tunnel barriers in the 2D layer in contact with borophene are zero, only graphene is $0.10 \mathrm{eV}$. Earlier findings have accorded that there is a tunnel barrier between the 2D material and bulk metal, so it can be seen that zero tunneling barrier is an irreplaceable favorable condition of borophene as a semiconductor contact layer.

The research on borophene is just beginning. With the development of the research on borophene, borophene not only has the above excellent properties but also may have novel atomic structure, excellent physical and chemical properties, and more interesting quantum effects, providing more possibilities for borophene-based applications in the future.

\section{Experimental Fabrications}

As is known to us all, a great challenge of fabricating borophene still exists due to the bonding configurations in bulk boron. Theoretically, a triangular lattice will be more stable if it has periodic holes $[53,65]$ and can grow on metal substrates, such as $\operatorname{Ag}(111)$ [66], $\mathrm{Au}(111)$ [67], and $\mathrm{Cu}(11)$ [68]. Owing to the metal passivation from stabilization of the $\mathrm{sp}^{2}$ hybridization and $\mathrm{Al}$ with the stabilization strategy of transferring one electron charge of Al to boron atom [69, 70], many researchers have synthesized atomically thin borophene via chemical vapor deposition (CVD), bottom-up 


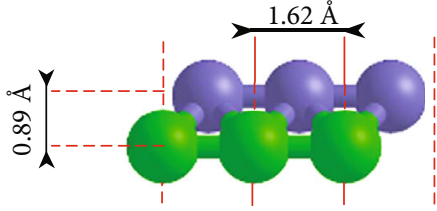

(a)

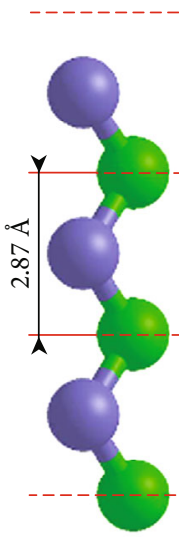

(b)

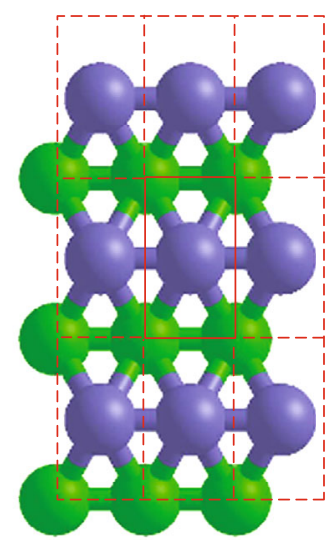

(c)

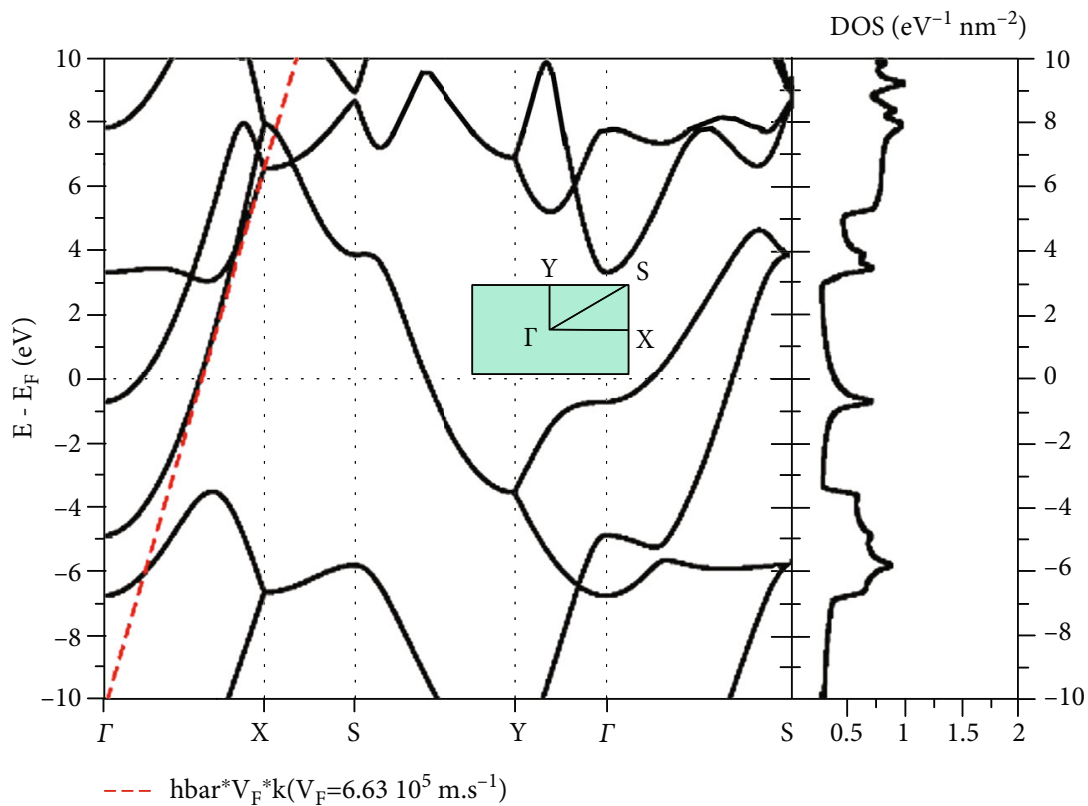

(d)

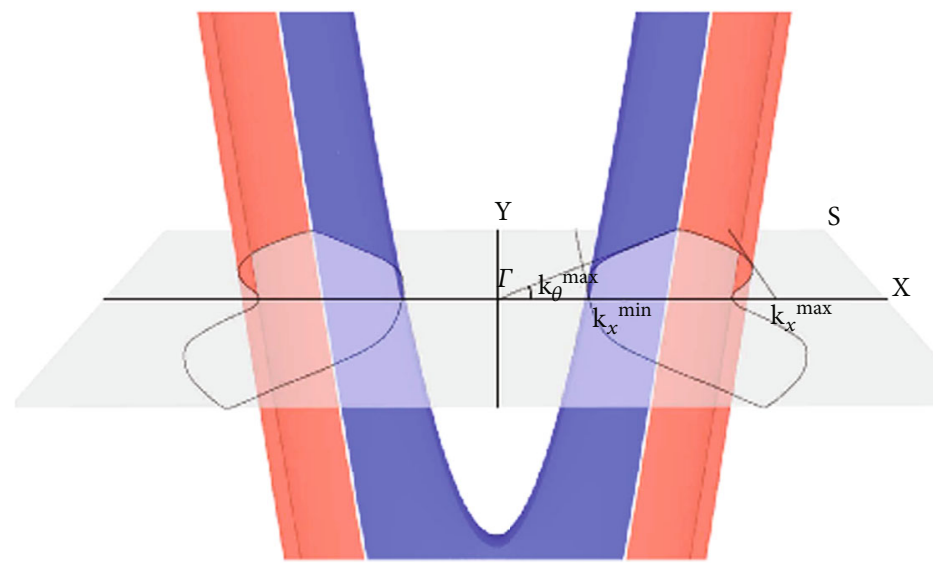

(e)

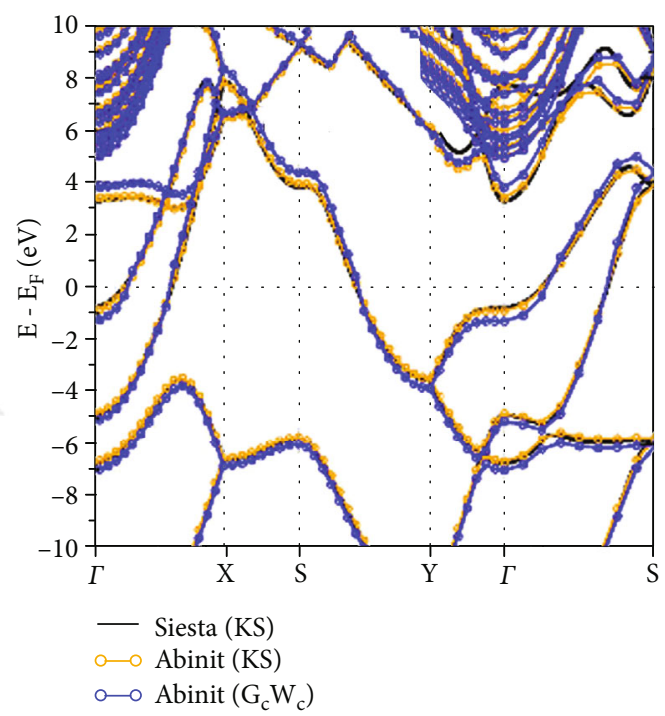

(f)

FIGURE 7: A $3 \times 3$ borophene super cell in (a, b) lateral views and (c) view from above. (d) DOS and electronic band structure with initial energy aligned to the EF. (e) 3D diagram of the two electronic energy bands crossing the EF in a TEW between $-1 \mathrm{ev}$ and $+1 \mathrm{eV}$. (f) The comparison between LDA with the $\mathrm{G}_{0} \mathrm{~W}_{0}$ and $\mathrm{KS}$ band structures with Siesta GGA-PBE amended one. Reprinted with permission from Ref. [47]. Copyright 2016 IOP Publishing.

fabrication techniques, top-down strategies, liquid-phase exfoliation, and sonochemical exfoliation techniques [25, $44,62,69,71,72]$. These rare fabrication investigations show the difficult exfoliation from nonlayer bulk B and thus seriously limit the further application of borophene.

\subsection{Bottom-Up Fabrication}

3.1.1. Physical Growth of Stripped Borophene. Mannix et al. firstly demonstrated the atomically thin borophene on the inert surface of $\operatorname{Ag}(111)$ with the morphology of striped- phase nanoribbon using molecular beam epitaxy (MBE) technique in Figures 9(a) and 9(b) [25]. Upon deposition at substrate temperature of $550^{\circ} \mathrm{C}$, the morphology shows a striped pattern in Figure 9(c) and a homogeneous pattern in Figure 9(d). The deposition rate and substrate temperature control the growth of these two phases. Figures 9(c) and 9(d) indicate the stable state of striped phase and metastable homogeneous phase. A parallel fabrication work by Feng et al. shows that borophene grows on a $\mathrm{Ag}(111)$ substrate epitaxial [71]. Figure 9(e) reveals the STM of a surface which is covered with $\beta_{12}$ borophene lumps. The atomic structure 


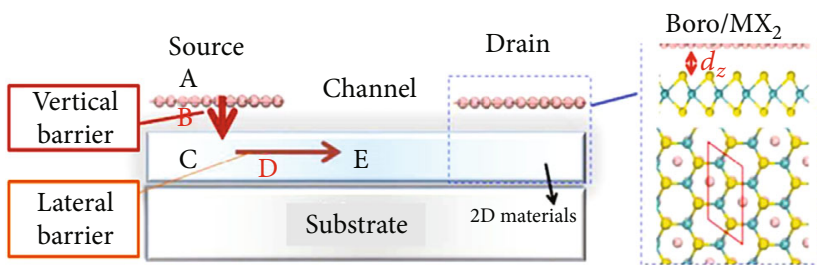

(a)

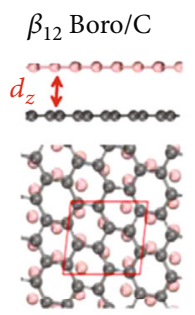

$\beta_{12}$ Boro/Si
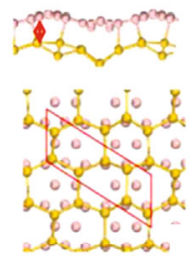

(c)
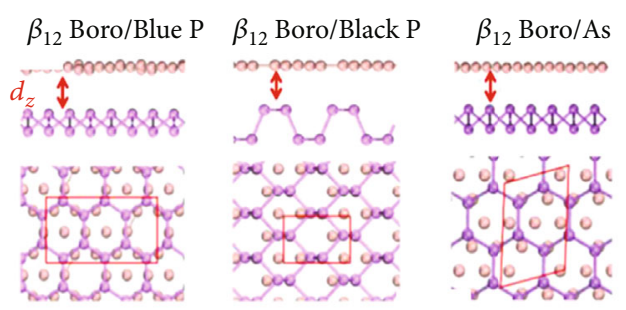

(d)

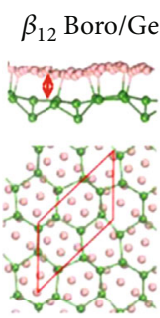

(b)

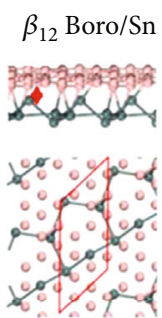

$\beta_{12} \mathrm{Boro} / \mathrm{Sb}$
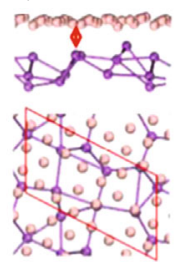
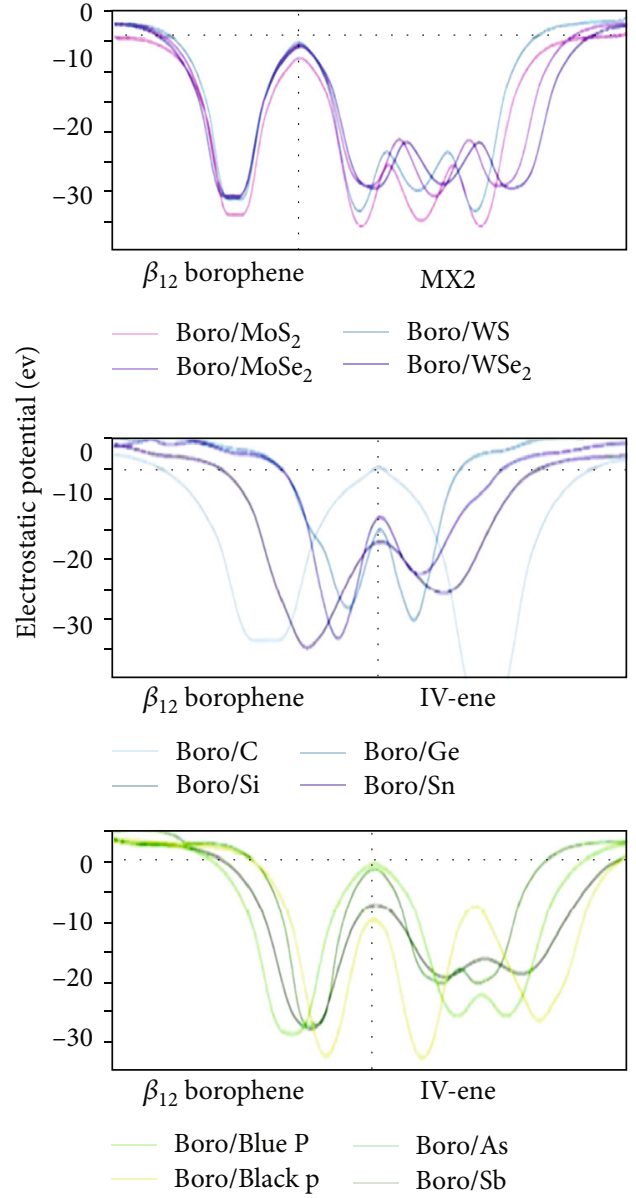

(e)

Figure 8: (a) $\beta_{12}$ borophene is in contact with 2D semiconductor atoms. The electron injection monolayer $\beta_{12}$ borophene shows its path $(\mathrm{A} \longrightarrow \mathrm{B} \longrightarrow \mathrm{C} \longrightarrow \mathrm{D} \longrightarrow \mathrm{E})$ by the red arrow. Lateral and top viewports of the utmost steady structure: (b) $\beta_{12}$ borophene gets to MX (M for Mo or W; X for S or Se); (c, d) $\beta_{12}$ borophene gets to the group IV-enes. (e) The balanced electrostatic potential with $z$ position for $\beta_{12}$ borophene/2D material interactions. Reprinted with permission from Ref. [34]. Copyright 2017 Royal Society of Chemistry.

diagram of $\beta_{12}$ borophene is rectangular [73]. Figure 9(f) reveals the STM topography and atomic structure model of $\chi 3$ borophene. The similar striped atomic arrangement can be obtained in both reported borophene in Figure 9(g).

Although the fabrication of borophene is successful, it is still unclear whether synthetic borophene can exist on structurally and chemically different layers. Boron's location in multiple chemical conditions is solved by sub-Angstrom spatial resolution, indicating that the borophene generates on planar layer which is larger than the Ag surface that is unreconstructed for about 2.4 A. It has the potential to develop wider diversity of $2 \mathrm{D}$ material through the separation from the growth substrate compared with the method of bulk layered crystal structures [74].

Different from the studied growth on Ag substrates, Kiraly et al. report that borophene islands can be generated under high temperatures with $\mathrm{Au}$ [75]. The method to produce borophene on $\mathrm{Au}(111)$ is different from the way that grows on Ag with only the surface (111). Importantly, the nucleation and growth of borophene are because of energy minimization and strain relief of the $\mathrm{Au}(111)$ surface. Owing to the increasing of boron coverage, the borophene changed from small well-organized islands to larger sheets, as shown in Figure 10(a). In Figures 10(b) and 10(c), the growth of one-atom thick boron islands has been proved in AFM.

The crystal structure is triangular with honeycomb lattice, and the exact ratio of honeycomb lattice sites and triangular sites is determined to be 1/5 in Figure 10(d). Furthermore, the first-principles calculations prove that the chargetransfer interaction occurs with minor degree of covalent bonding between borophene and $\mathrm{Cu}$. This study opens a bright future for borophene-based device fabrication.

3.1.2. Physical Growth of Honeycomb Borophene. Due to the planar honeycomb structure with sp2 hybridization in graphene, it makes it suitable for numerous promising applications [77], which leads to the research enthusiasm on other elemental 2D materials, for instance, silicene [78], germanene [79], and stanene [80]. However, these 2D materials are easy to form buckled honeycomb structure owing to the mixed sp2-sp3 hybridization as compared with sp2 hybridization in graphene with planar honeycomb structure. 


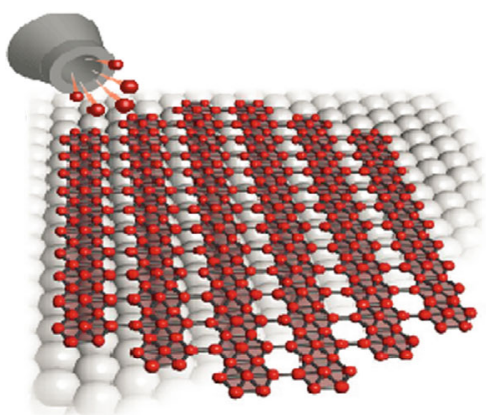

(a)

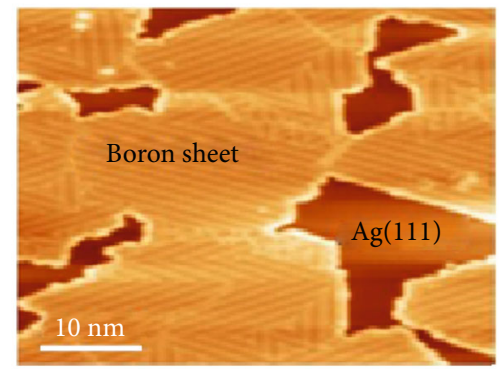

(e)

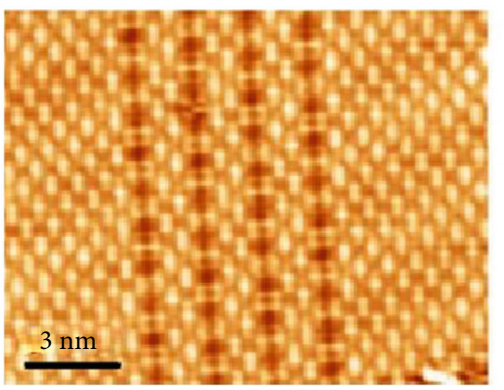

(f)
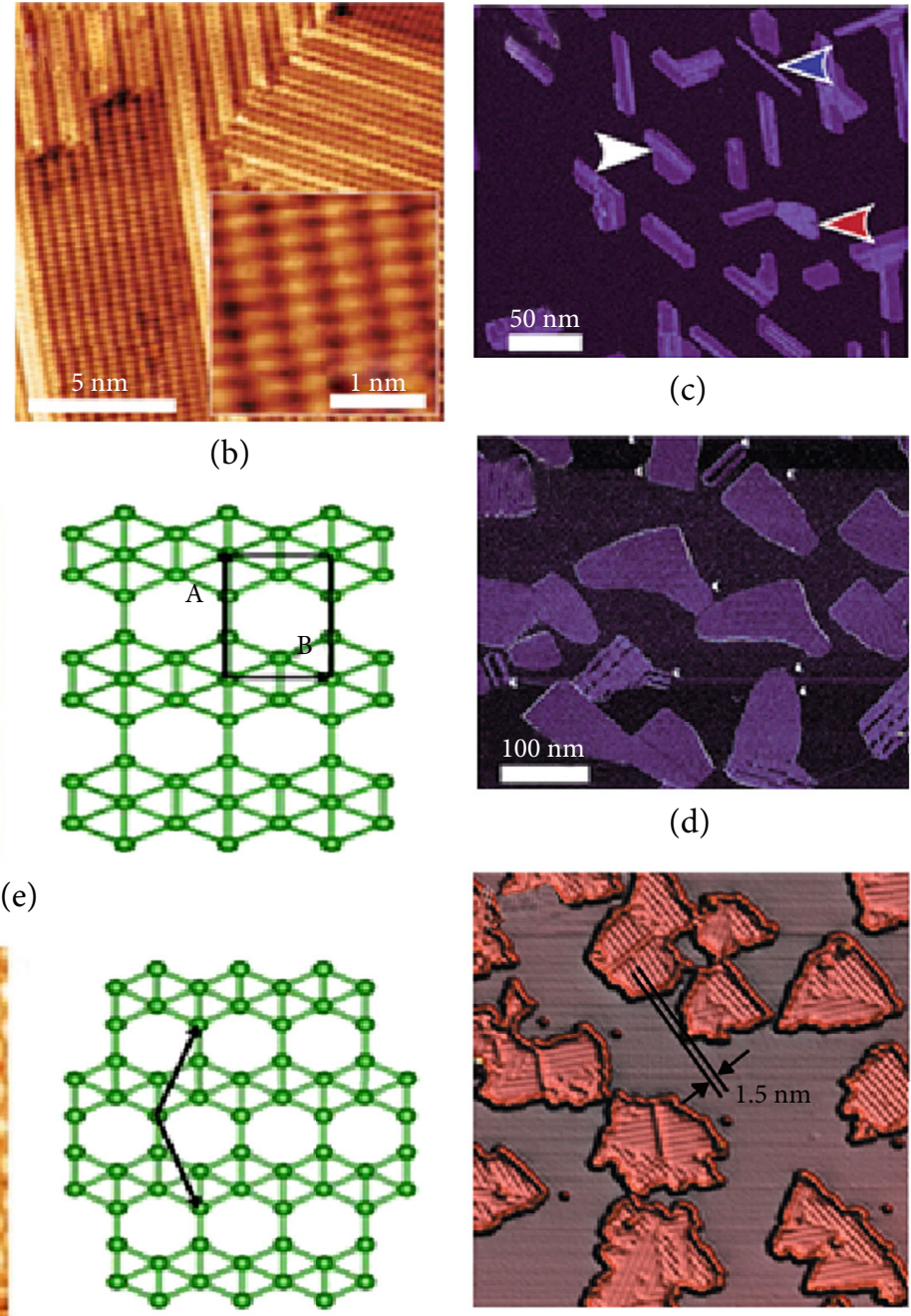

(c)

(b)
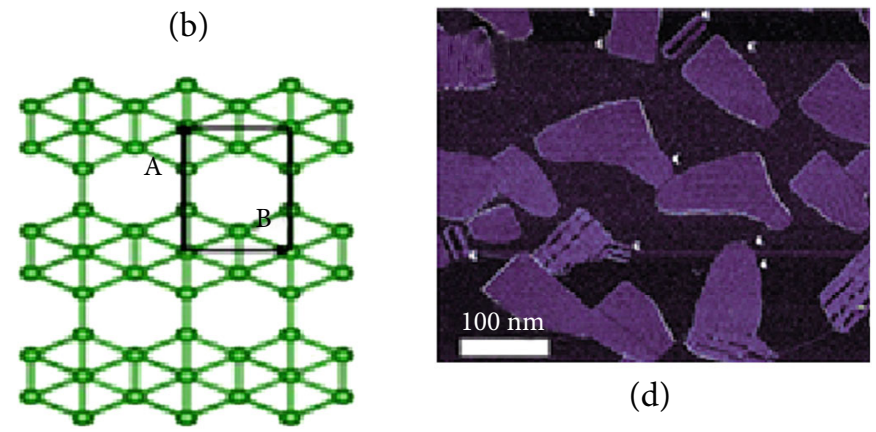

(d)

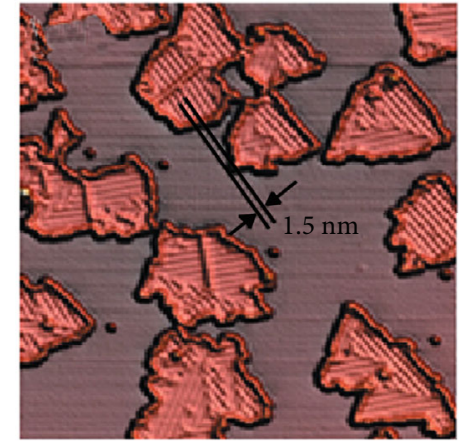

(g)

FIGURE 9: (a) Schematics of borophene growth. (b) STM topography pictures displaying striped-phase atomic-scale structure. (c) The sheets with striped phase and (d) homogeneous phase. Reprinted with permission from Ref. [25]. Copyright 2015 American Association for the Advancement of Science. (e) STM topography of $\beta_{12}$ borophene, the atomic structure diagram of $\beta_{12}$ borophene. (f) STM topography and atomic structure model of $\chi 3$ borophene. Reprinted with permission from Ref. [73]. Copyright 2019 American Chemical Society. (g) 3D STM topographic image. Reprinted with permission from Ref. [71]. Copyright 2016 Springer Nature Limited.

Fortunately, boron holds an even smaller atomic radius than carbon, rendering the possibility of forming planar honeycomb borophene.

Making the honeycomb borophene charming has three reasons. Firstly, in honeycomb lattice, the Dirac fermions have the similar electronic properties with group IV monoelemental-enes [81, 82]. Secondly, the honeycomb boron layer exists in $\mathrm{MgB}_{2}$ and it is just because of the honeycomb borophene that endows the high $T_{c}$ superconduction [83]. Extraction of the honeycomb borophene would further optimize the superconductivity $[49,84]$. Last but importantly, boron atom possesses three valence electrons, endowing the electron deficiency in honeycomb borophene and thus the unstable nature, which happens to satisfy the biodegradability need for biomedical applications.

The electron deficiency of boron makes honeycomb borophene challenging to be fabricated [69]. The honeycomb borophene can steadily exist in boride compounds (e.g., $\mathrm{MgB}_{2}$ ), which is because of the supplied electron for boron atoms from $\mathrm{Mg}$ atoms [85].

$\mathrm{Li}$ et al. successfully obtained the purely honeycomb, graphene-like borophene, through the way of using the $\mathrm{Al}(111)$ surface as the substrate; in the meantime, the molecular beam epitaxy (MBE) was also used which is grown in ultrahigh vacuum [69]. STM images apparently show the faultless monolayer borophene without buckled, planar honeycomb lattice alike as graphene in Figures 11(a) and 11(b). The line profile corresponding to the black line in Figure 11(a) is shown in Figure 11(c); the line profile corresponding to the green line in Figure 11(b) is shown in Figure $11(\mathrm{~d})$. The honeycomb borophene on $\mathrm{Al}(111)$ is energetically firm which can be obtained by the theoretical calculations. It is worth noting that almost one electron charge is shifted to every boron atom from the borophene/Al(111) 


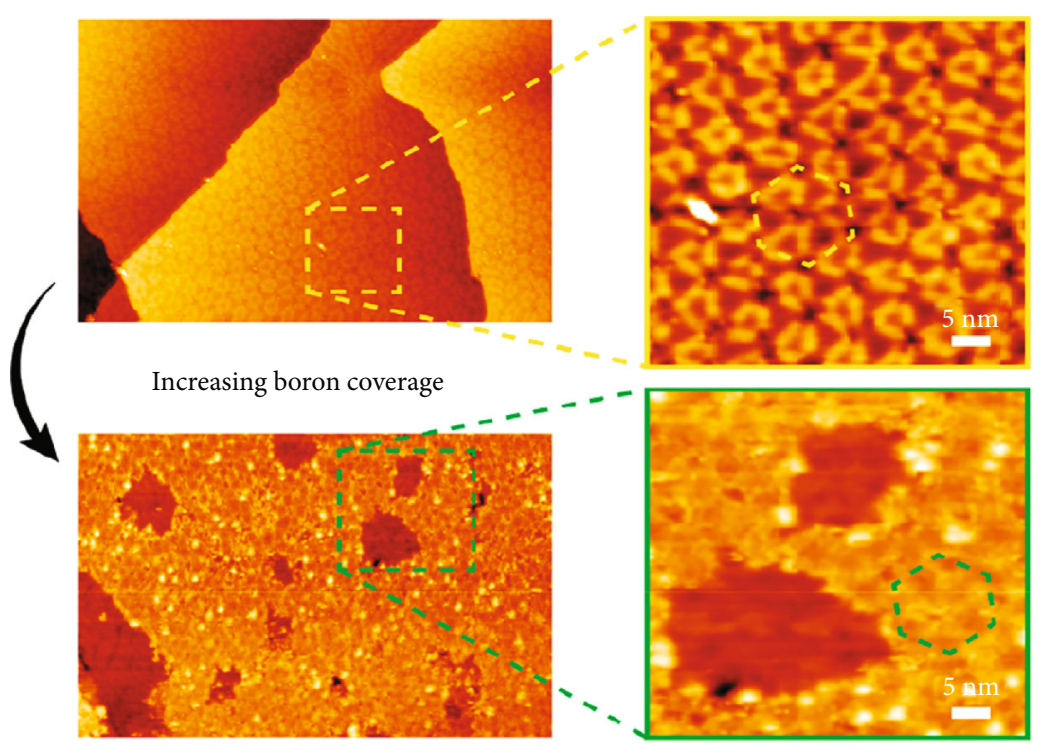

(a)

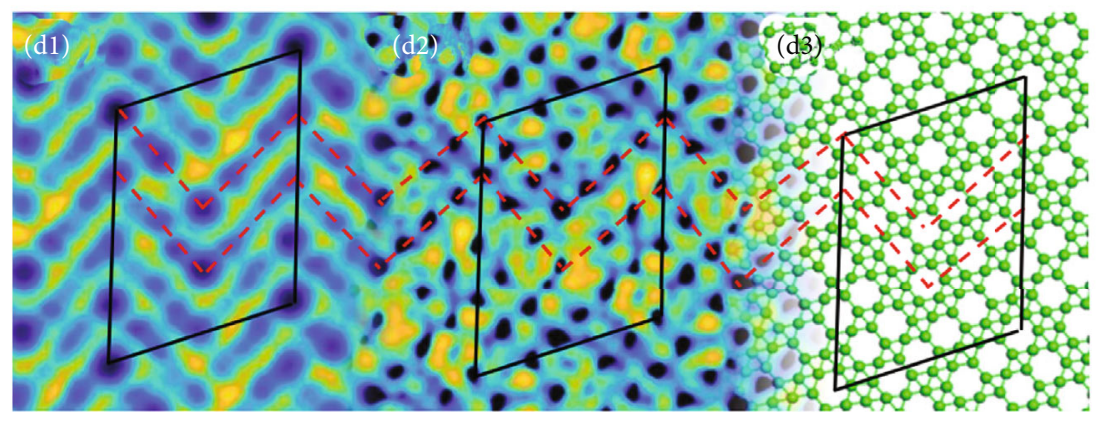

(d)

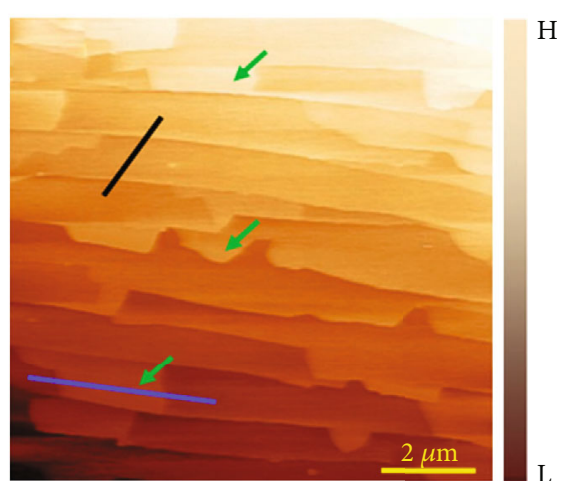

(b)
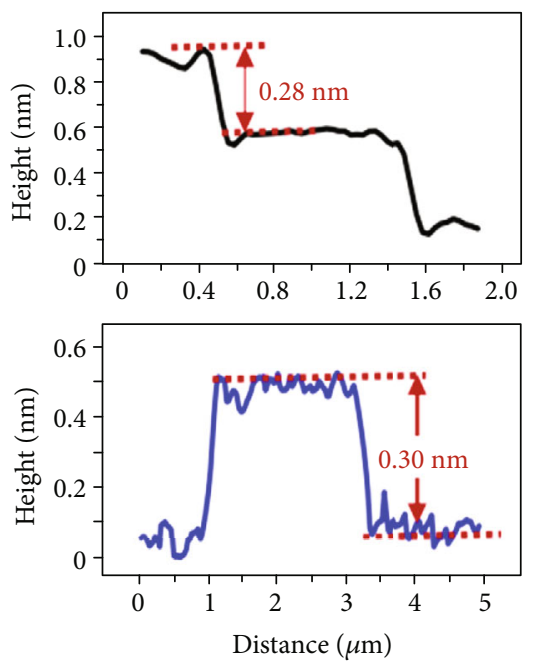

(c)

FIGURE 10: (a) Increasing boron dose leads to the breakdown of the network and growth of greater borophene islands. Reprinted with permission from Ref. [75]. Copyright 2019 American Chemical Society. (b) Topographic AFM image. (c) The line profile reveals a $2.8 \AA$ tall atomic step of the $\mathrm{Cu}$ substrate (the black line in (b)). And the line profile reveals that the thickness of the borophene sheet in surrounding conditions is about $3.0 \AA$ (the blue line in (b)). (d1) High-resolution STM data of borophene. (d2) DFT-imitated constant tunneling current isosurface of the proposed borophene structure. (d3) The diagram of the borophene structure with boron atoms and bonds shown in green. Reprinted with permission from Ref. [76]. Copyright 2019 Springer Nature Limited.

and steadies the honeycomb borophene structure; however, almost no charge was transferred in borophene/ $\operatorname{Ag}(111)$ in Figures 11(e) and 11(f). The existence of honeycomb 2D allotrope can play an import role on the basic study of boron chemistry; it can also supply a meaningful platform with which boron-based materials can be fabricated.

Zhu et al. comprehensively studied the properties of honeycomb borophene, such as the structural and energetic properties, that held on $\mathrm{Al}(111)$ by calculations [70]. Their calculations show a fierce bond energy between the honeycomb borophene and the $\mathrm{Al}(111)$ substrate; compared with the research before which indicated the coactions between graphene and some transition-metal surfaces, it is enormously stronger. The fierce coactions can be illustrated through the charge and back donation and the solid covalent bonding coactions between the honeycomb borophene and the substrate. The strong bond interaction contributed a lot to the stabilization of honeycomb borophene.
3.1.3. CVD Growth. Besides the physical growth, Tai et al. synthesized the borophene on $\mathrm{Cu}$ foils by chemical vapor deposition (CVD), as shown in Figure 12(a) [44]. Through elaborately designing the CVD furnace, the condition of the source region (T1) and the growth substrate region (T2) can be independently manipulated. The temperature of the $\mathrm{T} 1$ zone was set to be $1100^{\circ} \mathrm{C}$ to obtain the growth vapor, and the temperature of the $\mathrm{T} 2$ zone was set to be $1000^{\circ} \mathrm{C}$ to anneal the $\mathrm{Cu}$ foil. The borophene consists of orthorhombic g-B28 cells, which include B2 dumbbells and icosahedral B12 components in Figures 12(b) and 12(c). Although the growth techniques are totally different from the physical vapor epitaxy, the striped phase is also observed in Figure 12(d).

3.2. Top-Down Fabrication. The research on another efficient fabrication technique, i.e., direct exfoliation, is rarely reported because boron exhibits complex bonding network, 


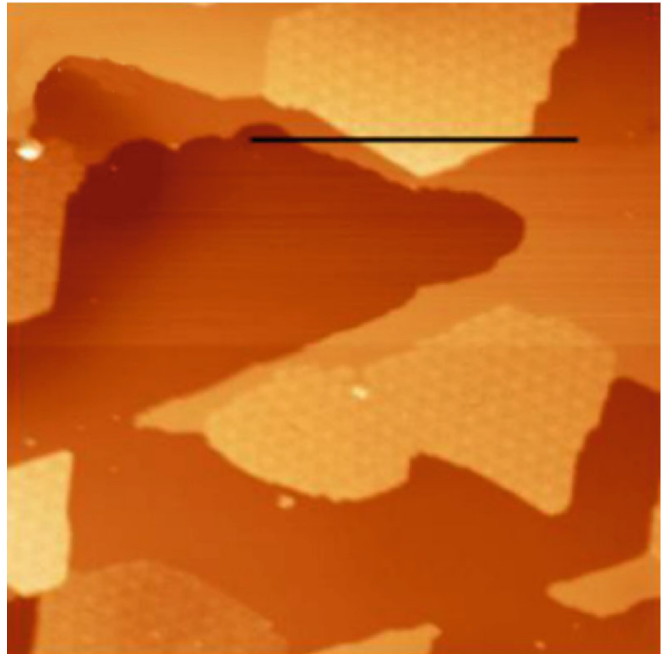

(a)

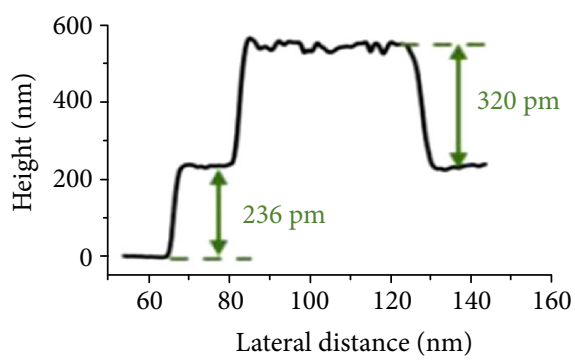

(c)
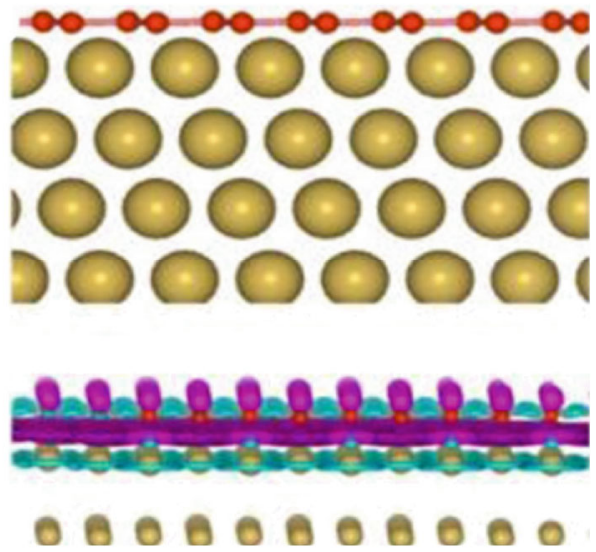

$\mathrm{B}$
$\mathrm{Al}$
$\mathrm{Ag}$

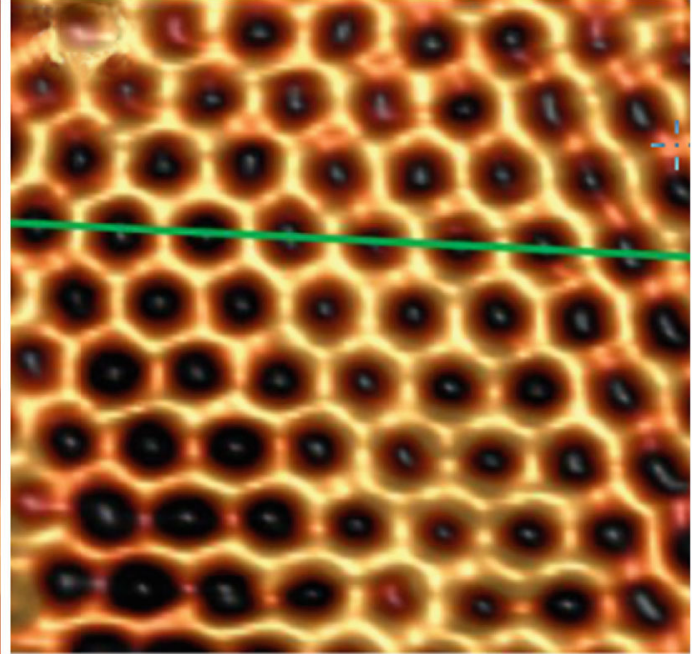

(b)

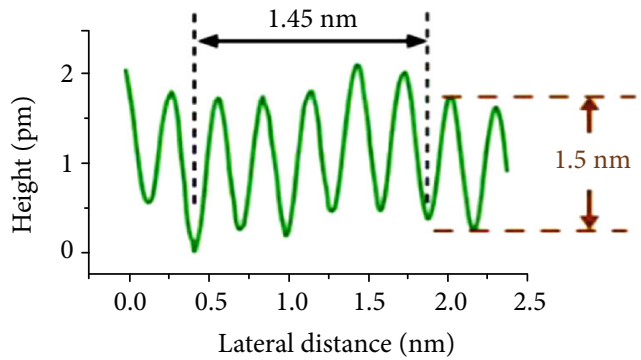

(d)

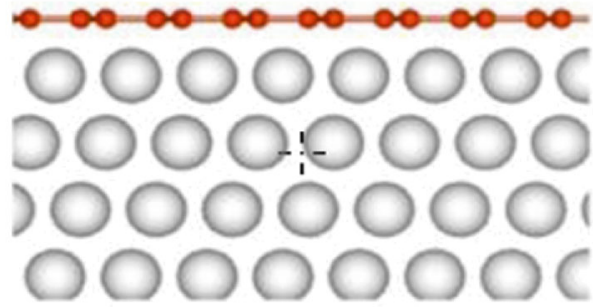

.8.8.8.8.8.8.8.8.8.8.8.8.

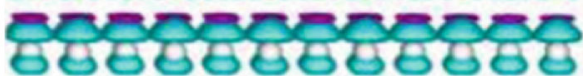

000000000000

(e)

FIGURE 11: (a) STM image showing a one-atom thick boron island running across an $\mathrm{Al}(111)$ step. (c) Line profile corresponding to the black line in (a). (b) The STM image of boron. (d) Line profile corresponding to the green line in (b). (e) Side elevation of honeycomb borophene on $\mathrm{Al}(111)$. (f) Side elevation of honeycomb borophene on $\mathrm{Ag}(111)$. Reprinted with permission from Ref. [69]. Copyright 2018 Science China Press.

rendering the direct exfoliation challenging. Ji et al. synthesized the borophene through a top-down approach, which can be divided into two times of liquid exfoliation and one thermal oxidation etching in Figure 13(a) [62]. After the first exfoliation, the $\mathrm{B}$ nanosheets appear to be thick. Then, the thick nanosheets can be oxidized to $\mathrm{B}_{2} \mathrm{O}_{3}$, which further dissolves into water. Later, the second ultrasonic exfoliation was employed. Through these three steps, the B nanosheets with 


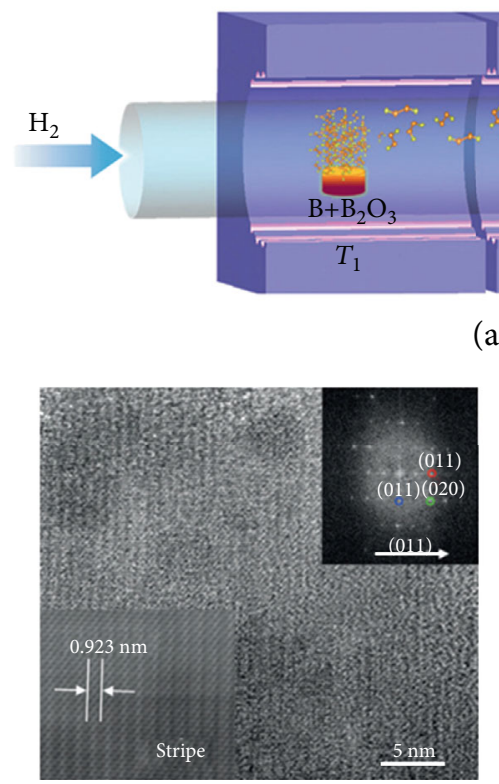

(d)

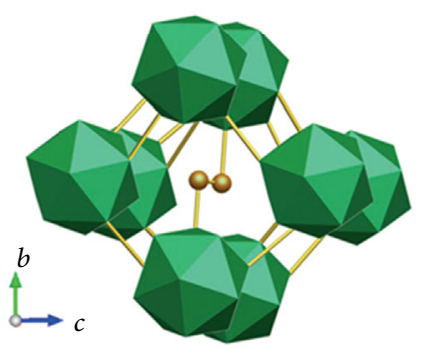

(c)

Figure 12: (a) Diagram of the homemade two-zone CVD furnace for growing borophene. (b) Top and lateral views of the borophene. (c) Atomic space structure of the elementary cell for borophene. (d) The striped phase shown by HRTEM image. Reprinted with permission from Ref. [44]. Copyright 2015 John Wiley \& Sons, Inc.

lateral size limit $100 \mathrm{~nm}$ and thickness less than $5 \mathrm{~nm}$ can be obtained (Figures 13(b)-13(d)). This fabrication strategy is fit for biomedical applications.

An easy and massive synthesis of atomic sheets of borophene through a fresh liquid-phase exfoliation and the reduction of borophene oxide is proved by Jiang et al. in Figure 13(e) [86]. Electron microscopy verified the existence of $\beta_{12}, \chi^{3}$, and their intermediate phases of borophene in Figure 13(f). These borophene materials and their hybrids will create great contributions in the realm of $2 \mathrm{D}$ materials and could contribute to develop future generations of apparatus and emerging applications.

The honeycomb borophene layer existing in diborides may also provide an avenue for obtaining honeycomb borophene directly through the top-down fabrication strategy $[87,88]$. However, the sandwiched boron layer is strongly bound with two external metal layers through covalent bond, endowing the direct exfoliation of honeycomb borophene a big challenge.

\section{Applications}

4.1. Energy Applications. Larger capacitance, good electrical conductivity, and ionic conductivity are the key to whether a material can be used as electrode material [89]. As the 2D material, borophene has high surface liveness, which is conducive to the realization of super high storage capacity of metal material. In addition, the metallic band construction of borophene facilitates the conduction of electricity, so it can also be used as the electrode for metal material ion batteries.

Because of the high surface activity of borophene, borophene as an electrode material has a strong interaction with lithium ion. The initial intercalation voltage is $1.12 \mathrm{eV}$, which reduces with the increase of Li adsorption range. Jiang et al. first proposed sodium borohydride as an excellent cathode material for lithium batteries [90]. The fully lithiated phase of 2-Pmmn is much higher than traditional graphite [90], silicene [91], phosphorene [92], and other electrode materials. As shown in Figure 14, the relative migration potential barrier of lithium ions in borophene is significantly smaller than that in graphite [90], silicene [92], phosphorene [91], and $\mathrm{Li}_{4} \mathrm{Ti}_{5} \mathrm{O}_{12}$, which is only $2.6 \mathrm{meV}$. The longitudinal potential barrier of borophene is about $325 \mathrm{meV}$, so the lithium ions on its surface have strong anisotropy during the migration process, and during the whole lithium process, the electronic structure of the lithium is characterized by the same characteristics of metal elements, indicating that borophene has good conductivity. To sum up, because borophene has high ionic conductivity and excellent electronic conductivity, it has relatively excellent performance in the whole charging and discharging process.

On the other hand, we can adjust properties of materials through doping, so as to realize the complementary advantages between materials [93]. Borophene hydride has a volume of $504 \mathrm{mAh} / \mathrm{g}$; borophene has a volume of $1860 \mathrm{mAh} / \mathrm{g}$; as a result, scientists in the study of borophene olefin as the anode of lithium-ion batteries when joined by hydrogen, due to the charge in the process of transfer, take borophene transfer to the hydrogen atom, so the borophene phase increases, thus reducing the interaction between lithium and borophene hydride. At the same time, the application of borophene in phases 12 and 3 in sodium and magnesium ion batteries also showed excellent characteristics. Lithium batteries using borophene in phases 12 and 3 as anode material were $1984 \mathrm{mAh} / \mathrm{g}$ and $1240 \mathrm{mAh} / \mathrm{g}$, which 


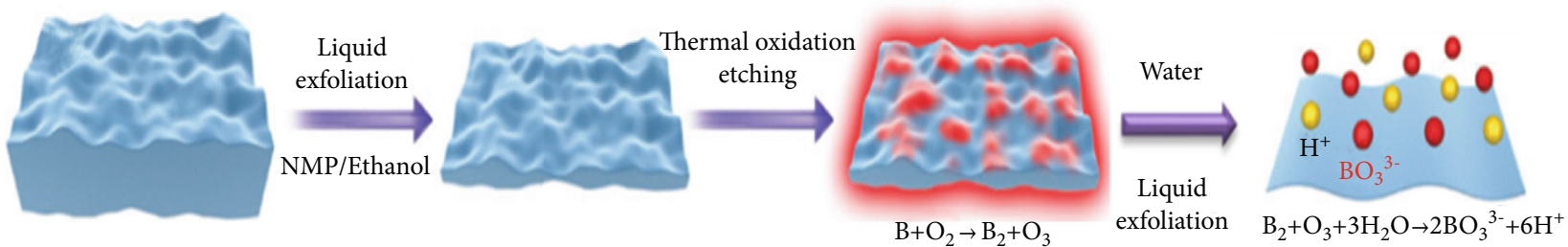

(a)

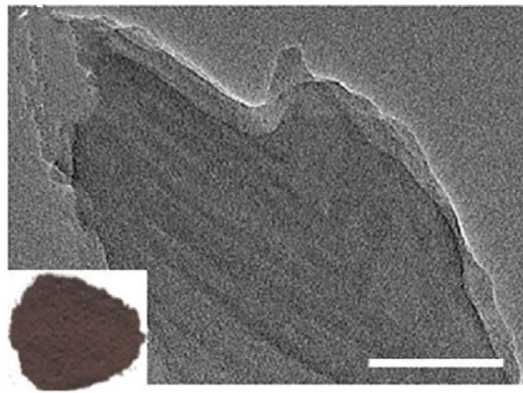

(b)

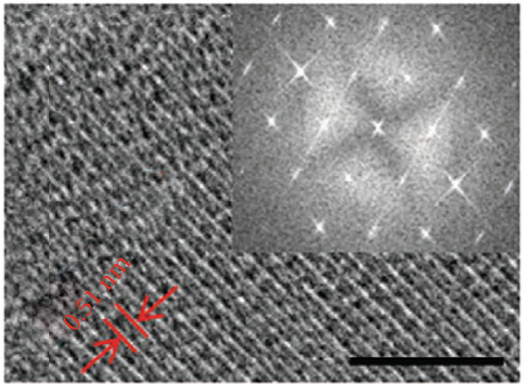

(c)

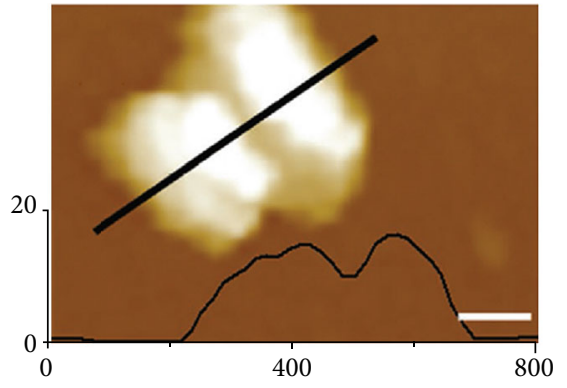

(d)

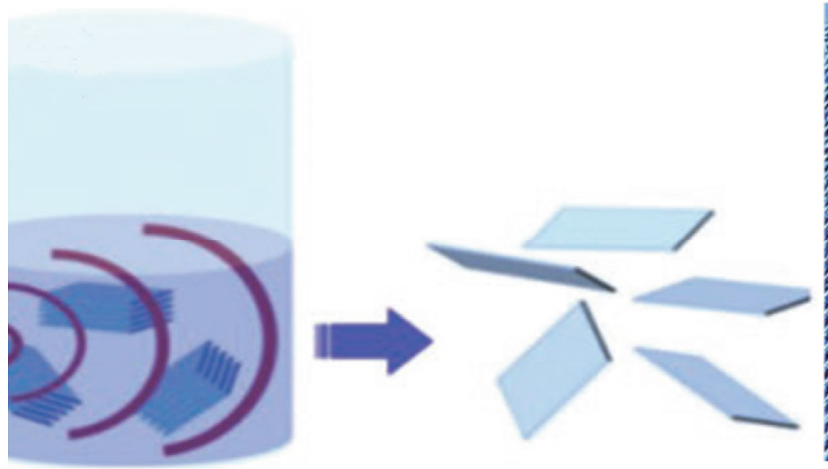

(e)

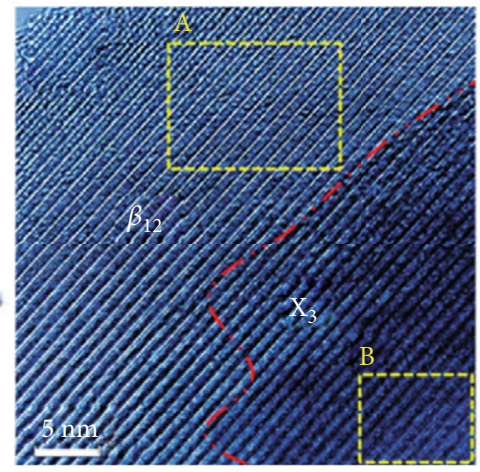

(f)

FIGURE 13: (a) Schematic representation of borophene. (b) HRTEM image, (c) TEM image, and (d) AFM image. Reprinted with permission from Ref. [62]. Copyright 2018 John Wiley \& Sons, Inc. (e) Diagrammatic drawing of liquid-phase exfoliation preparation of borophene. (f) HRTEM of borophene sheet with two phases. Reprinted with permission from Ref. [86]. Copyright 2018 Royal Society of Chemistry.

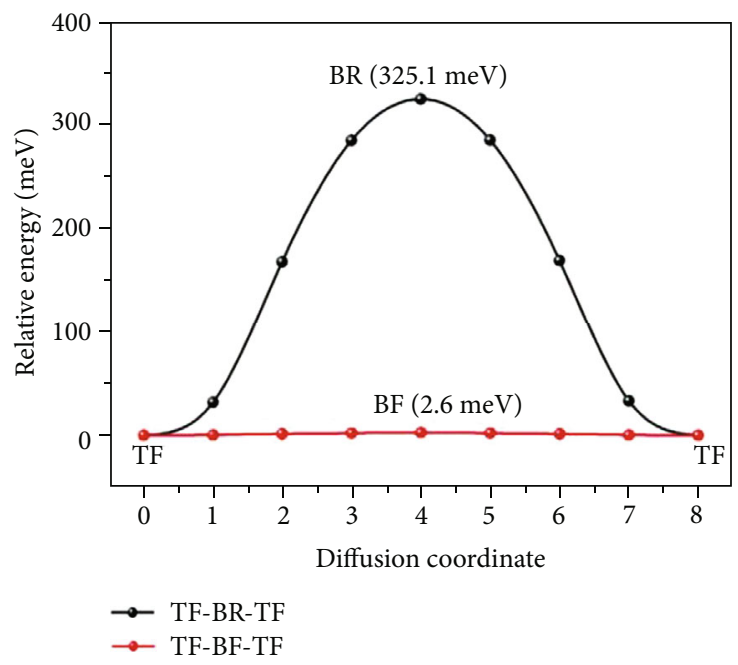

(a)

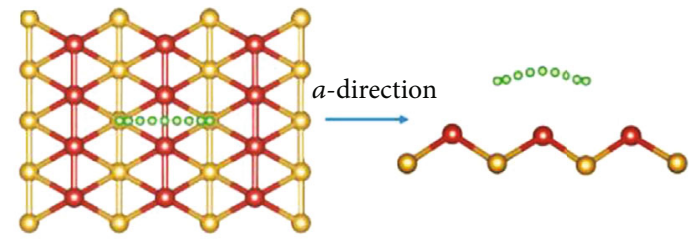

(b)
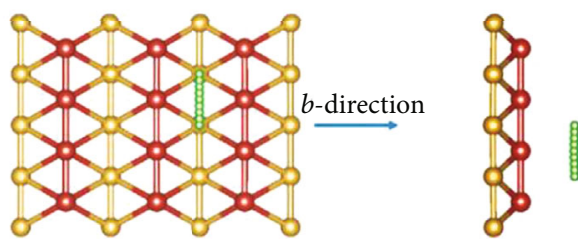

(c)

FIGURE 14: (a) Energy barriers and (b, c) pathways of Li movement alongside serrate and armchair directions. Reproduced from Ref. [90]. Copyright 2016 Elsevier B.V. 
TABLE 1: The adsorption energies (in eV) of $\mathrm{Li}_{2} \mathrm{~S}, \mathrm{Li}_{2} \mathrm{~S}_{2}, \mathrm{Li}_{2} \mathrm{~S}_{4}, \mathrm{Li}_{2} \mathrm{~S}_{6}$, and $\mathrm{Li}_{2} \mathrm{~S}_{8}$ on 2-Pmmn, $\chi 3, \beta_{12}$ borophene, graphene, and phosphorene [86, 100]. Reproduced from Ref. [99]. Copyright 2019 Springer Science.

\begin{tabular}{lcccccc}
\hline Species & $\mathrm{Li}_{2} \mathrm{~S}$ & $\mathrm{Li}_{2} \mathrm{~S}_{2}$ & $\mathrm{Li}_{2} \mathrm{~S}_{4}$ & $\mathrm{Li}_{2} \mathrm{~S}_{6}$ & $\mathrm{Li}_{2} \mathrm{~S}_{8}$ & Ref. \\
\hline 2-Pmmn & & & 6.45 & 4.32 & 6.18 & {$[90]$} \\
$\chi^{3}$ & & & 2.67 & 2.53 & 2.87 & {$[90]$} \\
$\beta_{12}$ & 3.34 & 2.89 & 1.45 & 1.53 & 1.36 & {$[101]$} \\
Graphene & & & 0.65 & 0.72 & 0.73 & {$[102]$} \\
Phosphorene & 2.51 & 1.91 & 1.27 & 1.00 & 1.12 & {$[102]$} \\
\hline
\end{tabular}

were far beyond traditional graphene, silicene, phosphorene, and $\mathrm{Li}_{4} \mathrm{Ti}_{5} \mathrm{O}_{12}$. The migration energy potential barrier of sodium borate ion in stage 12 is $330 \mathrm{meV}$, which is lower than that of lithium ion $(660 \mathrm{meV})$. The migration energy barrier of sodium borate at phase 3 was much lower than that of lithium at $660 \mathrm{meV}$. It can be seen from these reference data that borophene containing borophene vacancy is used as the cathode material of sodium ion battery, which has higher performance than traditional lithium ion battery in all aspects.

To sum up, theoretically, borophene has a high capacity, excellent conductivity, and efficient ion transport capacity, providing a broader prospect for the development of cathode materials for lithium, sodium, and magnesium ion batteries [94-98].

In recent years, the development of lithium-sulfur batteries has been seriously hindered; however, the right sulfur anchoring material can inhibit the shuttle effect [99]. The interaction between the lithium polysulfide and the anchoring material should be just right. As shown in Table 1, for graphene, the adsorption energies are $0.65 \mathrm{eV}$ and $0.72 \mathrm{eV}$, respectively, for $\mathrm{Li}_{2} \mathrm{~S}_{4}, \mathrm{Li}_{2} \mathrm{~S}_{6}$, and $\mathrm{Li}_{2} \mathrm{~S}_{8}$. Because of such interactions, graphene cannot be used as an anchoring material for polysulfide.

Zhao et al. have explored the application of borophene benzene as a potential anchoring material for lithium-sulfur batteries, using a first-principles calculation method [86, $103,104]$. The adsorption energies on the $2-\mathrm{Pmmn}$ phase of borophene benzene are $6.45,4.32$, and $6.18 \mathrm{eV}$, respectively, for $\mathrm{Li}_{2} \mathrm{~S}_{4}, \mathrm{Li}_{2} \mathrm{~S}_{6}$, and $\mathrm{Li}_{2} \mathrm{~S}_{8}$. Lithium polysulfide will be decomposed, and irreversible sulfur loss will occur due to interaction during absorbing and releasing electrical energy. Surprisingly, the mutual effect between lithium polysulfide and borophene benzene $\chi 3$ phase is much smaller than that between lithium polysulfide and borophene benzene 2Pmmn phase. The energies of lithium $\mathrm{Li}_{2} \mathrm{~S}_{4}$, lithium $\mathrm{Li}_{2} \mathrm{~S}_{6}$, and lithium $\mathrm{Li}_{2} \mathrm{~S}_{8}$ on the borophene benzene $\chi 3$ phase are $2.67,2.53$, and $2.87 \mathrm{eV}$, respectively, indicating that the borophene benzene $\chi^{3}$ phase is a perfect fixing material and can be used in lithium sulfur batteries. During the charging and discharging process of the battery, the appropriate adsorption strength is beneficial to inhibit the adsorption of spindle carbon on the electrode and protect its cycling structure from decomposition. The three phases of the borophene are a useful grappling material for batteries because of the metal structure characteristic of the borophene during the whole battery charging and discharging cycle. On the other hand, the borophene in phase 12 also showed good feature as a fixing material for lithium batteries. Moreover, the borophene of the $\beta_{12}$ also exhibits metallic properties throughout the battery cycle. Thus, $\chi^{3}$ and $\beta_{12}$ are promising anchoring materials for lithium batteries.

\subsection{Optoelectronic Applications}

\subsubsection{Sensor Application}

(1) Gas Sensor. Conventional carbon nanotubes and graphene gas sensors are highly sensitive to several irritating gases, but highly toxic chemicals such as formaldehyde cannot be identified. In recent years, the wide application prospect of borophene in gas sensors has attracted extensive attention [101].

In recent years, based on the DFT, researchers have studied the application prospect of borophene nanometer as formaldehyde sensor [101]. Through calculation and reasoning analysis, when $\mathrm{HCOH}$ molecule exists, the conductivity of borophene increases significantly, thus generating electrical signals. With the adsorption of more formaldehyde molecules by borophene, the strength of electrical signals increases, indicating that the sensitivity of borophene to formaldehyde gas is relatively high. In addition, borophene has high surface volume ratios and can be used to detect low-concentration gas molecules [102].

Huang et al. which studied in recent years have been able to succeed with bending and linear defects of preparation phase of the 2D borophene command on the NO gas molecular adsorption ability, by bending test and linear borophene in the adsorption NO gas after the $i-v$ characteristic curve, as shown in Figures 15(a) and 15(b); we can clearly see the sensitivity of the borophene to $\mathrm{NO}$ on electrical properties [102].

In addition, the borophene nanosheet also adsorbs ethanol. The adsorption of nanostructure borophene on oxygen atoms and hydrogen atoms on ethanol molecules is shown in Figures 15(c) and 15(d), which is at positions 1 and 2, respectively. Due to the adsorption of borophene and oxygen atoms and hydrogen atoms on ethanol, the bandgap of nanoborophene decreases rapidly and the conductivity increases. Therefore, the changes of adsorption energy and bandgap can be used to detect ethanol vapor.

4.2.2. The Electrocatalytic Applications. In practical applications, ideal gas adsorbents cannot meet the test requirements because of their weak adsorbability when adsorbing gas, nor can they affect the release after adsorption because of their strong adsorbability. Therefore, it is a great challenge to choose a material with appropriate adsorbability and good selectivity.

By using density functional theory (DFT) calculation method, extra electrons to the adsorbent are added. Tan et al. show that the borophene with negative charge on $\mathrm{CO}_{2}$ adsorption is more than before; in the experimental condition to achieve $\mathrm{CO}_{2}$ saturation coverage, adsorption amount has reached $6.73 \times 10^{-14} \mathrm{~cm}$ to $2 \times 10^{-14} \mathrm{~cm}[105,106]$. 


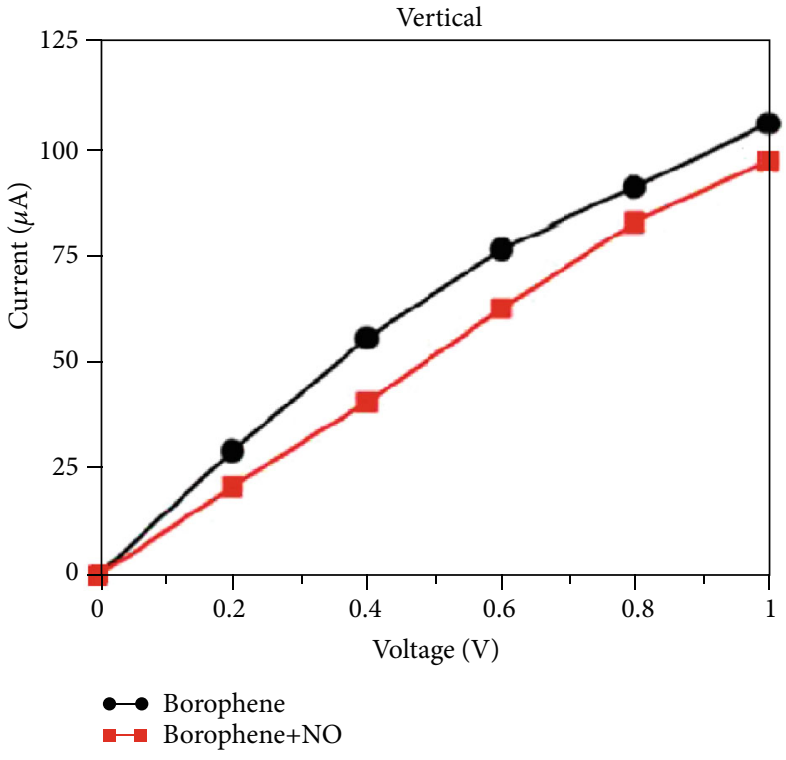

(a)

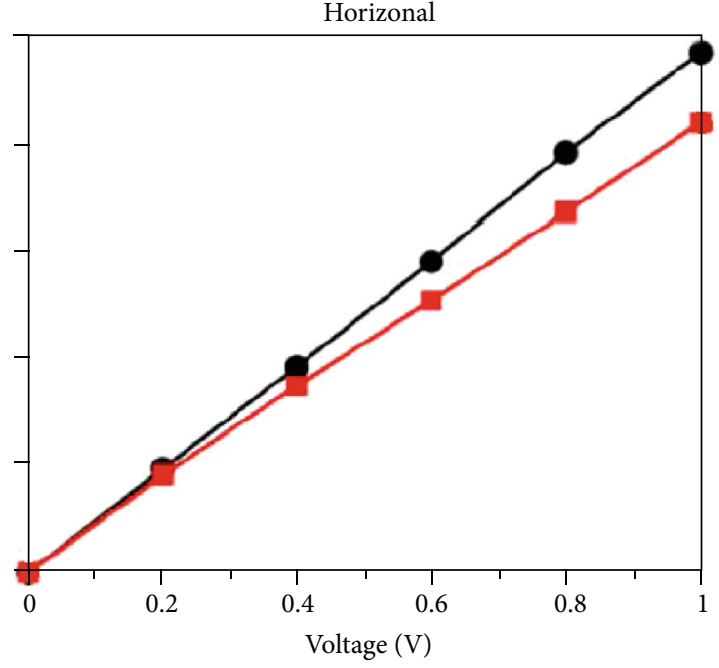

(b)

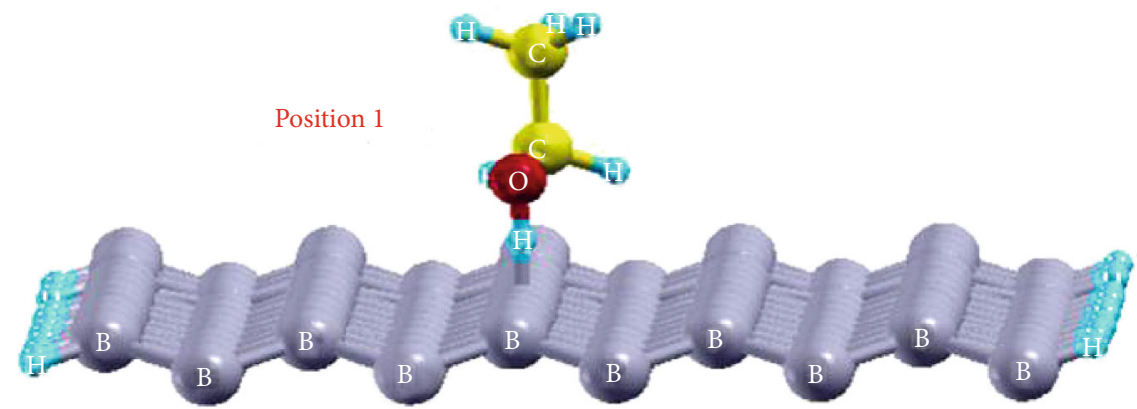

(c)

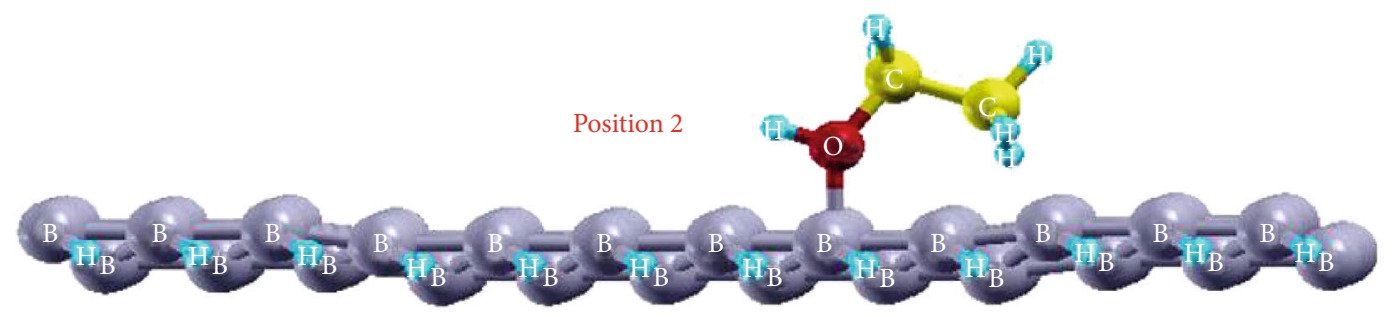

(d)

FIGURE 15: Current-voltage characteristics of borophene with and without adsorption of NO. The current flows in (a) vertical and (b) horizontal. Reprinted with permission from Ref. [101]. Copyright 2018 Journal of Physical Chemistry C. (c) Adsorption of ethanol molecules on position 1 and position 2. Reprinted with permission from Ref. [102]. Copyright 2017 Elsevier B.V.

On the other hand, other materials cannot release $\mathrm{CO}_{2}$ effectively after absorbing it, while the process of absorbing and releasing $\mathrm{CO}_{2}$ of negatively charged borophene is reversible, because we can control the absorption and release of borophene by controlling whether it is negatively charged, as shown in Figure 16(a), so as to realize electrical catalytic function.

As shown in Figure 16(b), the sensitivity of $\mathrm{CO}_{2}$ to charge density in the region of small negative charge density is much less than that in the region of large negative charge density. And adsorption on carbon and borophene in borophene associated with negative charge, when the borophene sug- gests that the negative charge increase, will transfer to $\mathrm{CO}_{2}$, so borophene above negative charge with $\mathrm{CO}_{2}$ above the repellency between the negative charges will also increase; therefore, in a small negative charge density of the area, more is absorbed.

\subsection{Biological Applications of Borophene}

4.3.1. Biomedical Applications. In recent years, PA imaging is a particularly promising biotechnology biophoton radiation diagnosis method because of its advantages of deep inspection, depth resolution 3D shadow, sensitive dexterity, stereo 


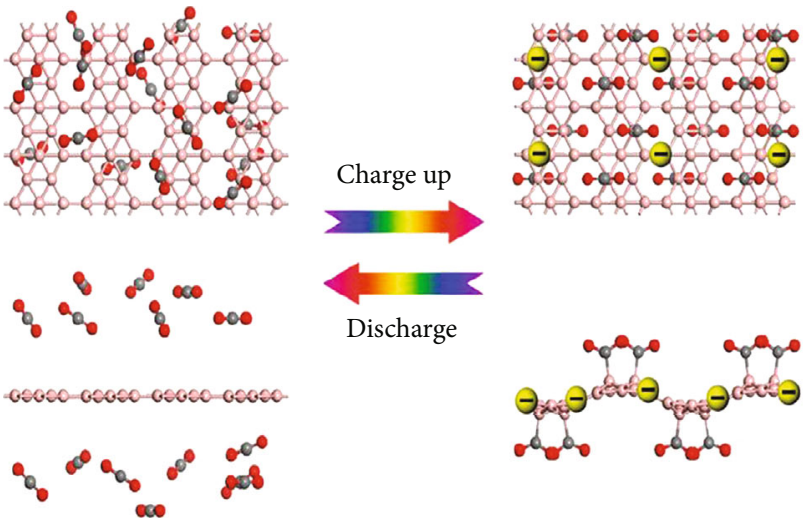

(a)

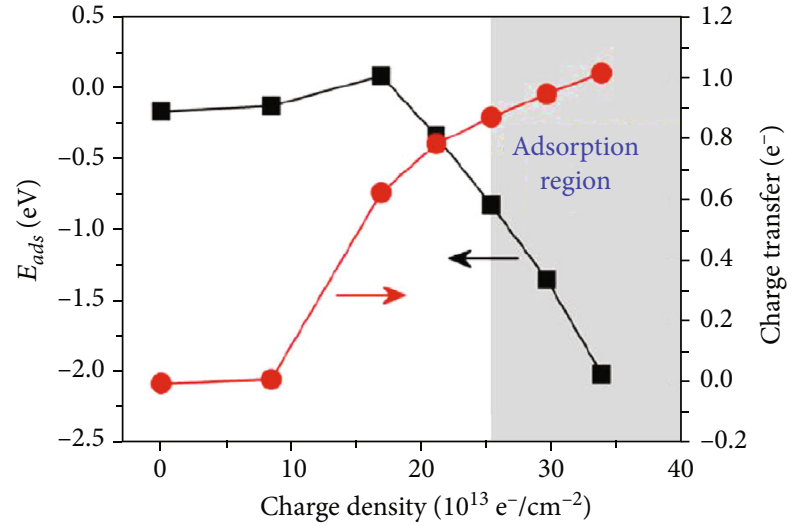

(b)

FIGURE 16: (a) $\mathrm{CO}_{2}$ molecules adsorbed negatively charged borophene more strongly after the addition of extra electrons to the adsorbent. (b) $\mathrm{CO}_{2}$ adsorption energy of borophene with extra electrons at $\mathrm{B}_{3}$ site and the charge transfer from borophene to $\mathrm{CO}_{2}$ molecule as functions of charge densities. The adsorption region is represented by the gray region. Reprinted with permission from Ref. [105]. Copyright 2017 American Chemical Society.

resolution, and image sharpness [107-109]. Because B-PEG NSs prepared by $2 \mathrm{D}$ borophene showed excellent photothermal performance, Ji et al. conducted in-depth research on the prospective of B-PEG NSs as PA carrier in mice [62].

Because of its excellent properties in depth resolution 3D shadow, sensitive dexterity, stereo resolution, and image sharpness, PA shadow has become one of the most promising biophotonic radiation diagnosis methods. Because of the excellent photothermal properties of B-PEG NSs in this study, the possibility of using B-PEG NSs as PA carrier in mice was experimentally investigated. As observed in Figure 17(a) with the increase of B-PEG NSs concentration, PA signal also becomes stronger and stronger. On the other hand, it can also be seen from Figure 17(c) that there is a good linear relationship between PA signal and B-PEG NSs concentration, so it can be inferred that B-PEG NSs is a good PA reagent. And as time went on, it was found that the PA signal became stronger and stronger, it is shown in Figure 17(b).

As shown in Figures 17(d) and 17(e), the changes in body temperature of mice in different treatment groups were recorded by infrared thermography, and it was found that the body temperature of mice in the group receiving near-infrared irradiation was significantly lower than that in the group without exposure. In addition, a large number of statistics and experiments have found that mice treated with chemotherapy-photothermal combination therapy showed extremely high tumor growth inhibition, indicating the good therapeutic effect of B-PEG/DOX NSs. As shown in Figure 17(f), the volume of tumor generated in mice can be clearly seen by comparison. The treatment has not found disturbing side effects such as abnormal weight loss (Figure 17(g)), eating, drinking, activity, or neurological problems.

4.3.2. Biosensor. In the field of biomedicine, borophene as a nanomaterial has been widely studied and paid attention to because of its good flexibility. This is because borophene has excellent molecular, physical, and mechanical properties in strip and tubular conformations.
In the biomedical applications of nanomaterials, borophene is often used as a $2 \mathrm{D}$ nanomaterial combined with other metals and semiconductor materials to improve the performance of biological applications without any negative effects [28]. DNA sequencing has been realized by different methods through the determination of base sequence, which has a great impact on the decoding of human biological code [110-112]. However, the application of nanomaterials in this technology will greatly accelerate the research of biology and medicine [82, 113-118].

Borophene is effectively used for biosensors. By testing the energy and electron sensitivity of $2 \mathrm{D}$ borophene to the four bases (adenine (A), guanine $(\mathrm{G})$, thymine $(\mathrm{T})$, and cytosine $(\mathrm{C})$ ) on top of biological DNA, the scientists found that different bases attached to borophene produce different conductivities, resulting in different electrical signals. In addition, Das et al. also measured the sensitivity of borophene to different bases, $A>G>C>T$ [119]. Thus, borophene nanosheets could be used in biological DNA sequencing devices.

Through the test and calculation, Rastgou et al. found that because the electronic sensitivity of borophene in different bases is different, the conductivity of borophene alkene will be different because of the action of different bases, as shown in Figure 18. In addition, the energy sensitivity of borophene alkene to different bases is also different, so the borophene alkene will have a broad application prospect in biological DNA sequencing [120].

Borophene looks more exciting than graphene. The "next generation of super nanomaterials" may be on the way. The borophene is expected to have a broader application and revolutionize energy, sensors, catalysts, and many other fields.

\section{Challenge and Perspectives}

This review systematically summarizes and classifies the recent developments of the borophene in terms of fabrications, properties, and various applications. We also provide our vital insights in the next discussions, aiming at placing 


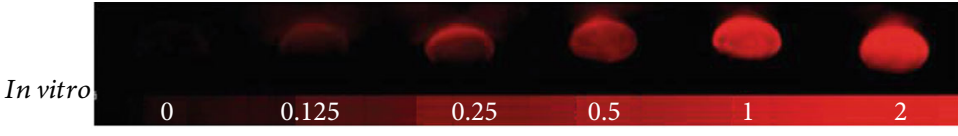

(a)
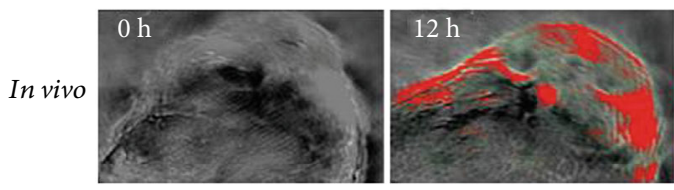

(b)

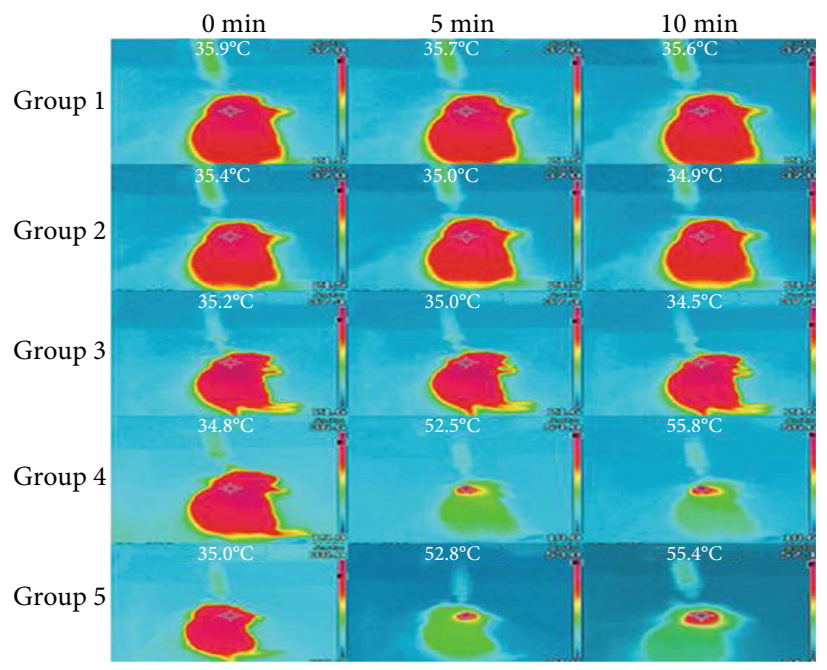

(d)

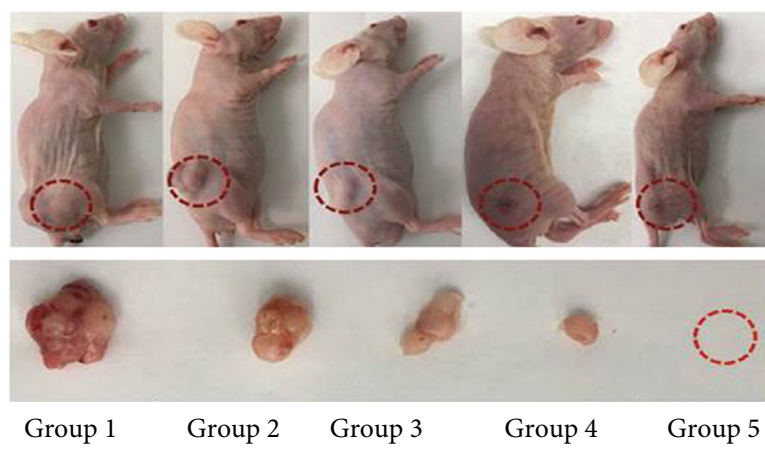

(f)

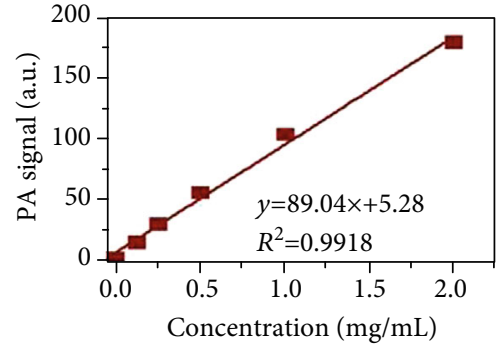

(c)

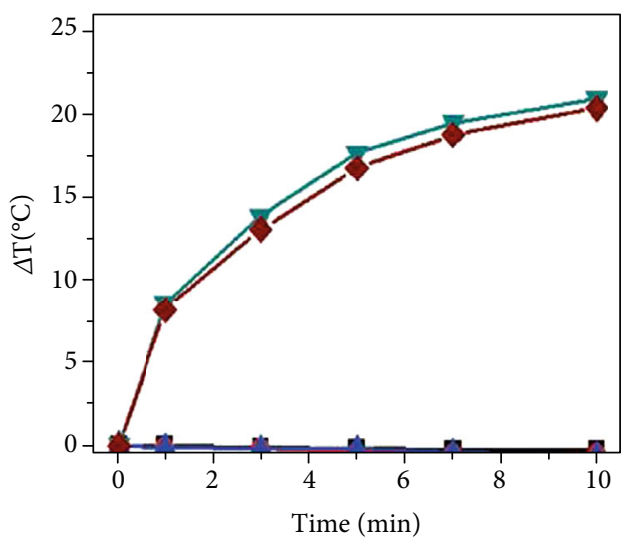

(e)

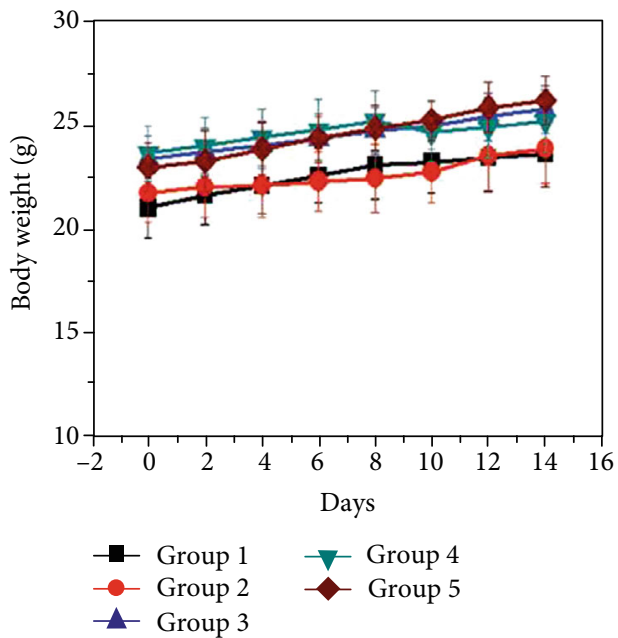

(g)

Figure 17: (a) In vitro PA pictures of using B-PEG NSs. (b) PA imaging of tumor sites over time (1, 12, and $24 \mathrm{~h})$ post injection. (c) PA values of using B-PEG NSs. (d) Infrared imaging. (e) Temperature changes with time in the MCF7 tumor-bearing mice after different handlings. (f) The tumor sites of each group were photographed after 14 days of treatment. (g) Changes in body weight were recorded during the experiment. Reprinted with permission from Ref. [62]. Copyright 2018 Wiley-Blackwell.

the emerging borophene in the pole position for energy, sensor, and biomedical applications.

The iterative feedback loop between theoretical and experimental results is widely considered to be the key to expediting the discovery and exploitation of new materials with desired properties. On the basis of theoretical research, the successful synthesis of borophene, along with the generation of other 2D crystal materials which have no layered bulk form, introduces a new way for the production of 2D materials by manipulating the intricate chemistry of materials.
The great consistency between theory and experimental practice for borophene indicates that further predictions are needed for future experimental investigations.

In the energy-related application of borophene, the good metal properties endow it to be a cathode material for extraordinary energy density in lithium batteries. Borophene also shows great potential in the application of biosensors. Borophene has different electrical sensitivities to different nucleobases, and it has been developed into a promising application in the sequencing of DNA of biological 

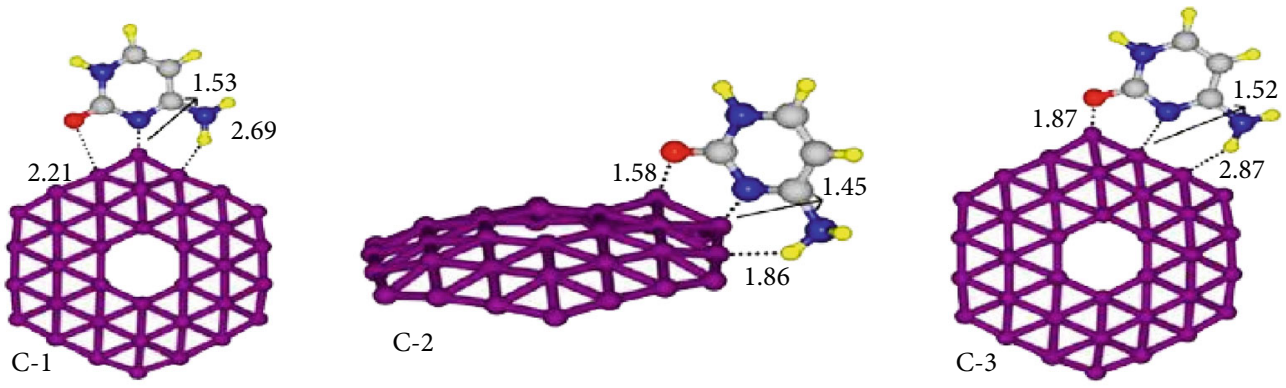

(a)

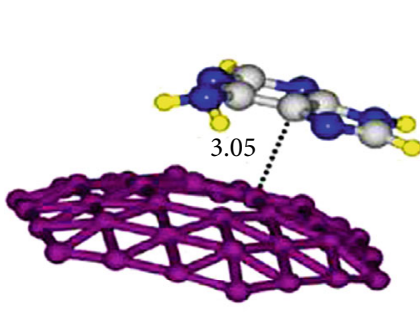

A-1
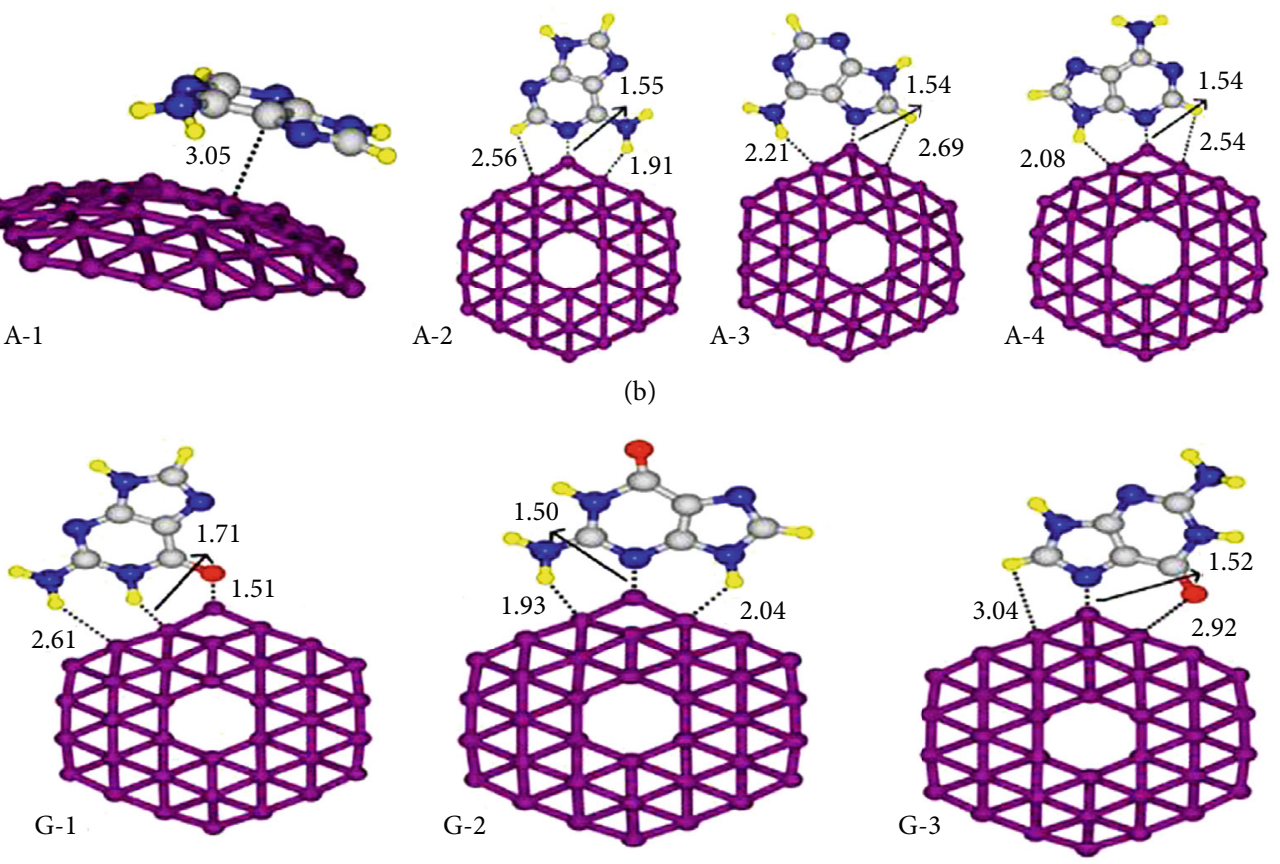

(c)
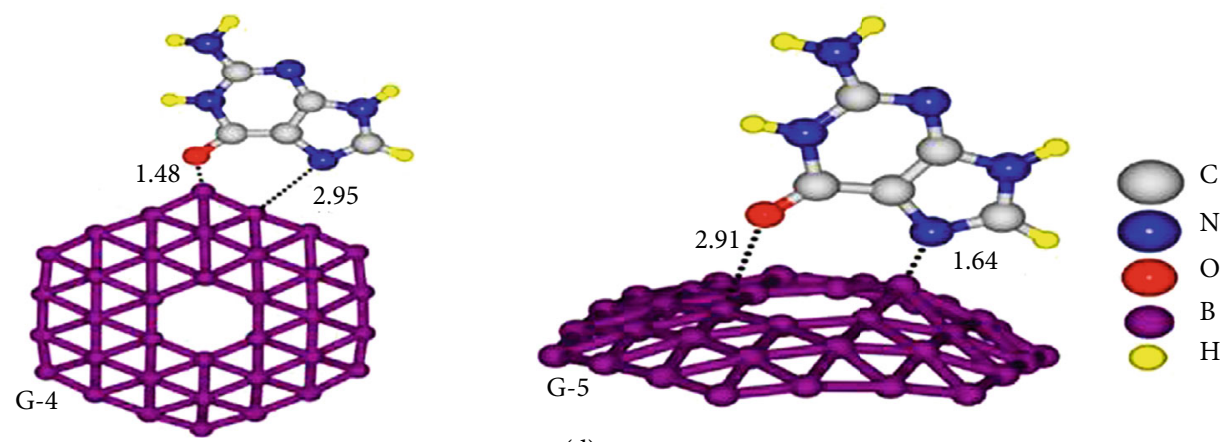

(d)

FIgURE 18: (a) Different adenines combine with borophene. Distances are in $\AA$. (b) Different adenines combine with borophene. Distances are in $\AA$. (c, d) Different adenines combine with borophene. Distances are in Å. Reprinted with permission from Ref. [120]. Copyright 2017 Elsevier.

genetic material, which may be a key step for humans to crack biological genetic code. In addition, when borophene is in contact with gas, its resistance varies greatly, which is promising for gas sensors. In terms of biological application, borophene has excellent biocompatibility but its NIR absorption needs to be further enhanced.

Although the properties and synthesis methods of borophene have been widely studied, the applications are still less.
Moreover, more theoretical work has been conducted but the experimental work is still.

At present, the synthesis of borophene suffers from low yield, and the borophene nanosheets are limited to be small sizes. In the future, the researchers need to improve the synthesis methods to achieve the large area and high-quality borophene nanosheets. Whether all-boron electronics could be realized in a manner similar to all-carbon electronics should 
be explored. Borophene applications can be extended from electronics to photovoltaic applications owing to its metallic nature. Most importantly, the low mechanical strength of borophene indicates its promising potential on wearable devices. For biomedical application, the Boron Neutron Capture Therapy (BNCT) can be further exploited by employing $2 \mathrm{D}$ borophene.

\section{Conflicts of Interest}

The authors declare that there are no conflicts of interest regarding the publication of this article.

\section{Authors' Contributions}

Zhongjian Xie, Xiangying Meng, and Xiangnan Li contributed equally to this work.

\section{Acknowledgments}

This work is supported by the State Key Research Development Program of China (Grant No. 2019YFB2203503), the National Natural Science Fund (Grant Nos. 61875138, 61435010 , and 61961136001), the Natural Science Foundation of Guangdong Province (Grant No. 2020A1515010612), and the Longhua District Science and Innovation Commission Project Grants (JCYJ201904) to Shenzhen International Institute for Biomedical Research.

\section{References}

[1] M. I. Katsnelson, "Graphene: carbon in two dimensions," Materials Today, vol. 10, no. 1-2, pp. 20-27, 2007.

[2] A. Gupta, T. Sakthivel, and S. Seal, "Recent development in 2D materials beyond graphene," Progress in Materials Science, vol. 73, pp. 44-126, 2015.

[3] H. Zhang, J. He, C. Zhai, and M. Zhu, " $2 \mathrm{D} \mathrm{Bi}_{2} \mathrm{WO}_{6} / \mathrm{MoS}_{2}$ as a new photo-activated carrier for boosting electrocatalytic methanol oxidation with visible light illumination," Chinese Chemical Letters, vol. 30, no. 12, pp. 2338-2342, 2019.

[4] B. Zheng, L. Qiao, H.-t. Yu, Q.-y. Wang, Y. Xie, and C.-q. Qu, "Enhanced field-emission properties of buckled $\alpha$-borophene by means of Li decoration: a first-principles investigation," Physical Chemistry Chemical Physics, vol. 20, no. 22, pp. 15139-15148, 2018.

[5] Q. H. Wang, K. Kalantar-Zadeh, A. Kis, J. N. Coleman, and M. S. Strano, "Electronics and optoelectronics of twodimensional transition metal dichalcogenides," Nature Nanotechnology, vol. 7, no. 11, pp. 699-712, 2012.

[6] C. Xing, Z. Xie, Z. Liang et al., "2D nonlayered selenium nanosheets: facile synthesis, photoluminescence, and ultrafast photonics," Advanced Optical Materials, vol. 5, no. 24, article 1700884, 2017.

[7] A. J. Mannix, Z. Zhang, N. P. Guisinger, B. I. Yakobson, and M. C. Hersam, "Borophene as a prototype for synthetic 2D materials development," Nature Nanotechnology, vol. 13, no. 6, pp. 444-450, 2018.

[8] T. Fan, Z. Xie, W. Huang, Z. Li, and H. Zhang, "Two-dimensional non-layered selenium nanoflakes: facile fabrications and applications for self-powered photo-detector," Nanotechnology, vol. 30, no. 11, p. 114002, 2019.
[9] Z. Xie, Y. P. Peng, L. Yu et al., "Solar-inspired water purification based on emerging 2D materials: status and challenges," Solar RRL, vol. 4, no. 3, article 1900400, 2020.

[10] Y. Zhou, M. Zhang, Z. Guo et al., "Recent advances in black phosphorus-based photonics, electronics, sensors and energy devices," Materials Horizons, vol. 4, no. 6, pp. 997-1019, 2017.

[11] G. Bhattacharyya, A. Mahata, I. Choudhuri, and B. Pathak, "Semiconducting phase in borophene: role of defect \& strain," Journal of Physics D Applied Physics, vol. 50, no. 40, p. $405103,2017$.

[12] Z. Xie, C. Xing, W. Huang et al., "Ultrathin 2D nonlayered tellurium nanosheets: facile liquid-phase exfoliation, characterization, and photoresponse with high performance and enhanced stability," Advanced Functional Materials, vol. 28, no. 16, article 1705833, 2018.

[13] Z.-Q. Wang, T.-Y. Lü, H.-Q. Wang, Y. P. Feng, and J.C. Zheng, "Review of borophene and its potential applications," Frontiers of Physics, vol. 14, no. 3, article 33403, 2019.

[14] Z. Xie, Y. Duo, Z. Lin et al., "The rise of 2D photothermal materials beyond graphene for clean water production," Advanced Science, vol. 7, no. 5, article 1902236, 2020.

[15] P. Li, Y. Chen, T. Yang et al., "Two-dimensional $\mathrm{CH} 3 \mathrm{NH} 3 \mathrm{PbI} 3$ perovskite nanosheets for ultrafast pulsed fiber lasers," ACS Applied Materials \& Interfaces, vol. 9, no. 14, pp. 12759-12765, 2017.

[16] J. Kwon and J. Kim, "Fabrication and properties of pndiodes with hybrid 2D layers: graphene/MoS2," Materials Express, vol. 8, no. 3, pp. 299-303, 2018.

[17] Z. Zhang, E. S. Penev, and B. I. Yakobson, "Two-dimensional boron: structures, properties and applications," Chemical Society Reviews, vol. 46, no. 22, pp. 6746-6763, 2017.

[18] S.-Y. Xie, Y. Wang, and X.-B. Li, "Flat boron: a new cousin of graphene," Advanced Materials, vol. 31, no. 36, article 1900392, 2019.

[19] W. Huang, Z. Xie, T. Fan et al., "Black-phosphorus-analogue tin monosulfide: an emerging optoelectronic twodimensional material for high-performance photodetection with improved stability under ambient/harsh conditions," Journal of Materials Chemistry C, vol. 6, no. 36, pp. 95829593, 2018.

[20] D. Golberg, Y. Bando, Y. Huang et al., "Boron nitride nanotubes and nanosheets," ACS Nano, vol. 4, no. 6, pp. 29792993, 2010.

[21] T. Ishii, T. Sato, Y. Sekikawa, and M. Iwata, "Growth of whiskers of hexagonal boron nitride," Journal of Crystal Growth, vol. 52, pp. 285-289, 1981.

[22] A. Nag, K. Raidongia, K. P. S. S. Hembram, R. Datta, U. V. Waghmare, and C. N. R. Rao, "Graphene analogues of BN: novel synthesis and properties," ACS Nano, vol. 4, no. 3, pp. 1539-1544, 2010.

[23] R. H. Wentorf, "Cubic form of boron nitride," Journal of Chemical Physics, vol. 26, no. 4, p. 956, 1957.

[24] J. H. Liao, Y. C. Zhao, Y. J. Zhao, H. Xu, and X. B. Yang, "Phonon-mediated superconductivity in $\mathrm{Mg}$ intercalated bilayer borophenes," Physical Chemistry Chemical Physics, vol. 19, no. 43, pp. 29237-29243, 2017.

[25] A. J. Mannix, X. F. Zhou, B. Kiraly et al., "Synthesis of borophenes: anisotropic, two-dimensional boron polymorphs," Science, vol. 350, no. 6267, pp. 1513-1516, 2015. 
[26] R. Peköz, M. Konuk, M. E. Kilic, and E. Durgun, "Twodimensional fluorinated boron sheets: mechanical, electronic, and thermal properties," Acs Omega, vol. 3, no. 2, pp. 1815$1822,2018$.

[27] V. V. Kulish, "Surface reactivity and vacancy defects in single-layer borophene polymorphs," Physical Chemistry Chemical Physics, vol. 19, no. 18, pp. 11273-11281, 2017.

[28] Z. A. Piazza, H.-S. Hu, W.-L. Li, Y.-F. Zhao, J. Li, and L.S. Wang, "Planar hexagonal $\mathrm{B}_{36}$ as a potential basis for extended single-atom layer boron sheets," Nature Communications, vol. 5, no. 1, article 3113, 2014.

[29] S. H. Mir, S. Chakraborty, P. C. Jha et al., "Two-dimensional boron: lightest catalyst for hydrogen and oxygen evolution reaction," Applied Physics Letters, vol. 109, no. 5, article 053903, 2016.

[30] Y. Zhao, S. Zeng, and J. Ni, "Superconductivity in twodimensional boron allotropes," Physical Review B, vol. 93, no. 1, article 014502, 2016.

[31] G. Qin, A. Du, and Q. Sun, "A theoretical insight into a feasible strategy for the fabrication of borophane," Physical Chemistry Chemical Physics, vol. 20, no. 23, pp. 16216-16221, 2018.

[32] Z. Zhang, Y. Yang, E. S. Penev, and B. I. Yakobson, "Elasticity, flexibility, and ideal strength of borophenes," Advanced Functional Materials, vol. 27, no. 9, article 1605059, 2017.

[33] L. Kong, L. Liu, L. Chen et al., "One-dimensional nearly free electron states in borophene," Nanoscale, vol. 11, no. 33, pp. 15605-15611, 2019.

[34] J. Yang, R. Quhe, S. Feng, Q. Zhang, M. Lei, and J. Lu, "Interfacial properties of borophene contacts with two-dimensional semiconductors," Physical Chemistry Chemical Physics, vol. 19, no. 35, pp. 23982-23989, 2017.

[35] B. Peng, H. Zhang, H. Shao, Y. Xu, R. Zhang, and H. Zhu, "The electronic, optical, and thermodynamic properties of borophene from first-principles calculations," Journal of Materials Chemistry C, vol. 4, no. 16, pp. 3592-3598, 2016.

[36] P. Krsti, "A computational study of hydrogen detection by borophene," Journal of Materials Chemistry C, vol. 5, no. 22, pp. 5426-5433, 2017.

[37] S. I. Vishkayi and M. B. Tagani, "Freestanding $\chi 3$-borophene nanoribbons: a density functional theory investigation," Physical Chemistry Chemical Physics, vol. 20, no. 15, pp. 10493-10501, 2018.

[38] Q. Zhao, Z. Xie, Y. P. Peng et al., "Current status and prospects of memristors based on novel 2D materials," Materials Horizons, 2020.

[39] X. Liu, L. Wang, S. Li, M. S. Rahn, B. I. Yakobson, and M. C. Hersam, "Geometric imaging of borophene polymorphs with functionalized probes," Nature Communications, vol. 10, no. 1, article 1642, 2019.

[40] D. Li, J. He, G. Ding et al., "Stretch-driven increase in ultrahigh thermal conductance of hydrogenated borophene and dimensionality crossover in phonon transmission," Advanced Functional Materials, vol. 28, no. 31, article 1801685, 2018.

[41] S. Verma, A. Mawrie, and T. K. Ghosh, "Effect of electronhole asymmetry on optical conductivity in $8-P m m n$ borophene," Physical Review B, vol. 96, no. 15, 2017.

[42] C. Xing, W. Huang, Z. Xie et al., "Ultrasmall bismuth quantum dots: facile liquid-phase exfoliation, characterization, and application in high-performance UV-Vis photodetector," ACS Photonics, vol. 5, no. 2, pp. 621-629, 2017.
[43] Y. Ge, Z. Zhu, Y. Xu et al., "Broadband nonlinear photoresponse of $2 \mathrm{D} \mathrm{TiS}_{2}$ for ultrashort pulse generation and alloptical thresholding devices," Advanced Optical Materials, vol. 6, no. 4, article 1701166, 2018.

[44] G. Tai, T. Hu, Y. Zhou et al., "Synthesis of atomically thin boron films on copper foils," Angewandte Chemie International Edition, vol. 54, no. 51, pp. 15473-15477, 2015.

[45] L. Adamska, S. Sadasivam, J. J. Foley IV, P. Darancet, and S. Sharifzadeh, "First-principles investigation of borophene as a monolayer transparent conductor," The Journal of Physical Chemistry C, vol. 122, no. 7, pp. 4037-4045, 2018.

[46] M. Tatullo, B. Zavan, F. Genovese et al., "Borophene is a promising 2D allotropic material for biomedical devices," Applied Sciences, vol. 9, no. 17, p. 3446, 2019.

[47] A. Lherbier, A. R. Botello-Méndez, and J.-C. Charlier, "Electronic and optical properties of pristine and oxidized borophene," 2D Materials, vol. 3, no. 4, article 045006, 2016.

[48] M. Ezawa, "Triplet fermions and Dirac fermions in borophene," Physical Review B, vol. 96, no. 3, article 035425, 2017.

[49] E. S. Penev, A. Kutana, and B. I. Yakobson, "Can twodimensional boron superconduct?," Nano Letters, vol. 16, no. 4, pp. 2522-2526, 2016.

[50] H. Zhong, K. Huang, G. Yu, and S. Yuan, "Electronic and mechanical properties of few-layer borophene," Physical Review B, vol. 98, no. 5, article 054104, 2018.

[51] C. Lian, S.-Q. Hu, J. Zhang et al., "Low-loss broadband Dirac plasmons in borophene," 2018, https://arxiv.org/abs/1803 .01604 .

[52] M. Jablan, H. Buljan, and M. Soljačić, "Plasmonics in graphene at infrared frequencies," Physical Review B, vol. 80, no. 24, article 245435, 2009.

[53] X. Yang, Y. Ding, and J. Ni, “Ab initio prediction of stable boron sheets and boron nanotubes: structure, stability, and electronic properties," Physical Review B, vol. 77, no. 4, article 041402, 2008.

[54] X. Liu, Z. Zhang, L. Wang, B. I. Yakobson, and M. C. Hersam, "Intermixing and periodic self-assembly of borophene line defects," Nature Materials, vol. 17, no. 9, pp. 783-788, 2018.

[55] J. Zeng and K. Chen, "Charge transport in borophene: role of intrinsic line defects," Journal of Physical Chemistry C, vol. 123, no. 10, pp. 6270-6275, 2019.

[56] Z. Sun, Y. Zhao, Z. Li et al., "TiL ${ }_{4}$-coordinated black phosphorus quantum dots as an efficient contrast agent for in vivo photoacoustic imaging of cancer," Small, vol. 13, no. 11, article 1602896, 2017.

[57] X. Zhen, J. Zhang, J. Huang, C. Xie, Q. Miao, and K. Pu, "Macrotheranostic probe with disease-activated nearinfrared fluorescence, photoacoustic, and photothermal signals for imaging-guided therapy," Angewandte Chemie International Edition, vol. 57, no. 26, pp. 7804-7808, 2018.

[58] Z. Xie, S. Chen, Y. Duo et al., "Biocompatible two-dimensional titanium nanosheets for multimodal imaging-guided cancer theranostics," ACS Applied Materials \& Interfaces, vol. 11, no. 25, pp. 22129-22140, 2019.

[59] B. Kang, D. Yu, Y. Dai, S. Chang, D. Chen, and Y. Ding, "Cancer-cell targeting and photoacoustic therapy using carbon nanotubes as "Bomb" agents," Small, vol. 5, no. 11, pp. 1292-1301, 2009.

[60] J. Chen, T. Fan, Z. Xie et al., "Advances in nanomaterials for photodynamic therapy applications: status and challenges," Biomaterials, vol. 237, article 119827, 2020. 
[61] B. Kang, Y. Dai, S. Chang, and D. Chen, "Explosion of singlewalled carbon nanotubes in suspension induced by a large photoacoustic effect," Carbon, vol. 46, no. 6, pp. 978-981, 2008.

[62] X. Ji, N. Kong, J. Wang et al., "A novel top-down synthesis of ultrathin 2D boron nanosheets for multimodal imagingguided cancer therapy," Advanced Materials, vol. 30, no. 36, article 1803031, 2018.

[63] Y. Huang, S. N. Shirodkar, and B. I. Yakobson, "Two-dimensional boron polymorphs for visible range plasmonics: a firstprinciples exploration," Journal of the American Chemical Society, vol. 139, no. 47, pp. 17181-17185, 2017.

[64] S. Das, H.-Y. Chen, A. V. Penumatcha, and J. Appenzelle, "High performance multilayer $\mathrm{MoS}_{2}$ transistors with scandium contacts," Nano Letters, vol. 13, no. 1, pp. 100-105, 2013.

[65] H. Tang and S. Ismail-Beigi, "Novel precursors for boron nanotubes: the competition of two-center and three-center bonding in boron sheets," Physical Review Letters, vol. 99, no. 11, article 115501, 2007.

[66] Q. Chen, W. J. Tian, L. Y. Feng et al., "Planar $\mathrm{B}_{38}-$ and $\mathrm{B}_{37}-$ clusters with a double-hexagonal vacancy: molecular motifs for borophenes," Nanoscale, vol. 9, no. 13, pp. 4550-4557, 2017.

[67] Y. Liu, E. S. Penev, and B. I. Yakobson, "Probing the synthesis of two-dimensional boron by first-principles computations," Angewandte Chemie International Edition, vol. 52, no. 11, pp. 3156-3159, 2013.

[68] H. Liu, J. Gao, and J. Zhao, "From boron cluster to twodimensional boron sheet on $\mathrm{Cu}(111)$ surface: growth mechanism and hole formation," Scientific Reports, vol. 3, no. 1, article 3238, 2013.

[69] W. Li, L. Kong, C. Chen et al., "Experimental realization of honeycomb borophene," Science Bulletin, vol. 63, no. 5, pp. 282-286, 2018.

[70] L. Zhu, B. Zhao, T. Zhang, G. Chen, and S. A. Yang, "How is honeycomb borophene stabilized on $\mathrm{Al}(111)$," The Journal of Physical Chemistry C, vol. 123, no. 23, pp. 14858-14864, 2019.

[71] B. Feng, J. Zhang, Q. Zhong et al., "Experimental realization of two-dimensional boron sheets," Nature Chemistry, vol. 8, no. 6, pp. 563-568, 2016.

[72] P. Ranjan, T. K. Sahu, R. Bhushan et al., "Freestanding borophene and its hybrids," Advanced Materials, vol. 31, no. 27, article 1900353, 2019.

[73] S. Sheng, J. B. Wu, X. Cong et al., "Raman spectroscopy of two-dimensional borophene sheets," ACS Nano, vol. 13, no. 4, pp. 4133-4139, 2019.

[74] G. P. Campbell, A. J. Mannix, J. D. Emery et al., "Resolving the chemically discrete structure of synthetic borophene polymorphs," Nano Letters, vol. 18, no. 5, pp. 2816-2821, 2018.

[75] B. Kiraly, X. Liu, L. Wang et al., "Borophene synthesis on Au (111)," ACS Nano, vol. 13, no. 4, pp. 3816-3822, 2019.

[76] R. Wu, I. K. Drozdov, S. Eltinge et al., "Large-area singlecrystal sheets of borophene on $\mathrm{Cu}$ (111) surfaces," Nature Nanotechnology, vol. 14, no. 1, pp. 44-49, 2019.

[77] Y. Yao, F. Ye, X. L. Qi, S. C. Zhang, and Z. Fang, "Spin-orbit gap of graphene: first-principles calculations," Physical Review B, vol. 75, no. 4, article 041401, 2007.

[78] J. Zhao, H. Liu, Z. Yu et al., "Rise of silicene: a competitive 2D material," Progress in Materials Science, vol. 83, pp. 24-151, 2016.
[79] A. Acun, L. Zhang, P. Bampoulis et al., "Germanene: the germanium analogue of graphene," Journal of Physics: Condensed Matter, vol. 27, no. 44, article 443002, 2015.

[80] F.-F. Zhu, W. J. Chen, Y. Xu et al., "Epitaxial growth of twodimensional stanene," Nature Materials, vol. 14, no. 10, pp. 1020-1025, 2015.

[81] Z. Meng, T. C. Lang, S. Wessel, F. F. Assaad, and A. Muramatsu, "Quantum spin liquid emerging in twodimensional correlated Dirac fermions," Nature, vol. 464, no. 7290 , pp. 847-851, 2010.

[82] A. M. Attaran, S. Abdol-Manafi, M. Javanbakht, and M. Enhessari, "Voltammetric sensor based on $\mathrm{Co}_{3} \mathrm{O}_{4} / \mathrm{SnO}_{2}$ nanopowders for determination of diltiazem in tablets and biological fluids," Journal of Nanostructure in Chemistry, vol. 6, no. 2, pp. 121-128, 2016.

[83] P. Canfield, D. K. Finnemore, S. L. Bud'ko et al., "Superconductivity in dense $\mathrm{MgB}_{2}$ wires," Physical Review Letters, vol. 86, no. 11, pp. 2423-2426, 2001.

[84] C. Cheng, J. T. Sun, H. Liu et al., "Suppressed superconductivity in substrate-supported $\beta 12$ borophene by tensile strain and electron doping," $2 D$ Materials, vol. 4, no. 2, article 025032, 2017.

[85] A. N. Kolmogorov and S. Curtarolo, "Prediction of different crystal structure phases in metal borides: a lithium monoboride analog to $\mathrm{MgB}_{2}$," Physical Review B, vol. 73 , no. 18 , article 180501, 2006.

[86] H. Jiang, W. Shyy, M. Liu, Y. X. Ren, and T. S. Zhao, "Borophene and defective borophene as potential anchoring materials for lithium-sulfur batteries: a first-principles study," Journal of Materials Chemistry A, vol. 6, no. 5, pp. 21072114, 2018.

[87] S. Carenco, D. Portehault, C. Boissière, N. Mézailles, and C. Sanchez, "Nanoscaled metal borides and phosphides: recent developments and perspectives," Chemical Reviews, vol. 113, no. 10, pp. 7981-8065, 2013.

[88] A. N. Kolmogorov and S. Curtarolo, "Theoretical study of metal borides stability," Physical Review B, vol. 74, no. 22, article 224507, 2006.

[89] D. Ma, Y. Li, H. Mi et al., "Robust $\mathrm{SnO} 2-x$ nanoparticleimpregnated carbon nanofibers with outstanding electrochemical performance for advanced sodium-ion batteries," Angewandte Chemie International Edition, vol. 57, no. 29, pp. 8901-8905, 2018.

[90] H. R. Jiang, Z. Lu, M. C. Wu, F. Ciucci, and T. S. Zhao, "Borophene: a promising anode material offering high specific capacity and high rate capability for lithium-ion batteries," Nano Energy, vol. 23, pp. 97-104, 2016.

[91] Q.-F. Li, C. G. Duan, X. G. Wan, and J. L. Kuo, “Theoretical prediction of anode materials in Li-ion batteries on layered black and blue phosphorus," The Journal of Physical Chemistry C, vol. 119, no. 16, pp. 8662-8670, 2015.

[92] G. A. Tritsaris, E. Kaxiras, S. Meng, and E. Wang, "Adsorption and diffusion of lithium on layered silicon for Li-ion storage," Nano Letters, vol. 13, no. 5, pp. 2258-2263, 2013.

[93] Z. Zhang, S. N. Shirodkar, Y. Yang, and B. I. Yakobson, "Gate-voltage control of borophene structure formation," Angewandte Chemie, vol. 129, no. 48, pp. 15623-15628, 2017.

[94] J. Liu, C. Zhang, L. Xu, and S. Ju, "Borophene as a promising anode material for sodium-ion batteries with high capacity and high rate capability using DFT," RSC Advances, vol. 8, no. 32, pp. 17773-17785, 2018. 
[95] X. Zhang, J. Hu, Y. Cheng, H. Y. Yang, Y. Yao, and S. A. Yang, "Borophene as an extremely high capacity electrode material for Li-ion and Na-ion batteries," Nanoscale, vol. 8, no. 33, pp. 15340-15347, 2016.

[96] S. Banerjee, G. Periyasamy, and S. K. Pati, "Possible application of 2D-boron sheets as anode material in lithium ion battery: a DFT and AIMD study," Journal of Materials Chemistry A, vol. 2, no. 11, pp. 3856-3864, 2014.

[97] L. Jia, X. Chen, X. Deng et al., "How to boost the sluggish lithium-ion hopping dynamic in borophene?," Applied Surface Science, vol. 441, pp. 356-363, 2018.

[98] P. Xiang, X. Chen, W. Zhang et al., "Metallic borophene polytypes as lightweight anode materials for non-lithium-ion batteries," Physical Chemistry Chemical Physics Pccp, vol. 19, no. 36, pp. 24945-24954, 2017.

[99] F. Li, Y. Su, and J. Zhao, "Shuttle inhibition by chemical adsorption of lithium polysulfides in B and $\mathrm{N}$ co-doped graphene for Li-S batteries," Physical Chemistry Chemical Physics, vol. 18, no. 36, pp. 25241-25248, 2016.

[100] S. P. Jand, Y. Chen, and P. Kaghazchi, "Comparative theoretical study of adsorption of lithium polysulfides $\left(\mathrm{Li}_{2} \mathrm{~S}_{\mathrm{x}}\right)$ on pristine and defective graphene," Journal of Power Sources, vol. 308, pp. 166-171, 2016.

[101] P. K. Kannan, D. J. Late, H. Morgan, and C. S. Rout, "Recent developments in 2D layered inorganic nanomaterials for sensing," Nanoscale, vol. 7, no. 32, pp. 13293-13312, 2015.

[102] C.-S. Huang, A. Murat, V. Babar, E. Montes, and U. Schwingenschlögl, "Adsorption of the gas molecules $\mathrm{NH}_{3}, \mathrm{NO}, \mathrm{NO}_{2}$, and $\mathrm{CO}$ on borophene," The Journal of Physical Chemistry C, vol. 122, no. 26, pp. 14665-14670, 2018.

[103] L. Zhang, P. Liang, H. B. Shu et al., "Borophene as efficient sulfur hosts for lithium-sulfur batteries: suppressing shuttle effect and improving conductivity," The Journal of Physical Chemistry C, vol. 121, no. 29, pp. 15549-15555, 2017.

[104] F. Li and J. Zhao, "Atomic sulfur anchored on silicene, phosphorene, and borophene for excellent cycle performance of Li-S batteries," ACS Applied Materials \& Interfaces, vol. 9, no. 49, pp. 42836-42844, 2017.

[105] X. Tan, H. A. Tahini, and S. C. Smith, "Borophene as a promising material for charge-modulated switchable $\mathrm{CO}_{2}$ capture," ACS Applied Materials \& Interfaces, vol. 9, no. 23, pp. 19825-19830, 2016.

[106] Q. Sun, Z. Li, D. J. Searles, Y. Chen, G. (. M.). Lu, and A. du, "Charge-controlled switchable $\mathrm{CO}_{2}$ capture on boron nitride nanomaterials," Journal of the American Chemical Society, vol. 135, no. 22, pp. 8246-8253, 2013.

[107] L. V. Wang and S. Hu, "Photoacoustic tomography: in vivo imaging from organelles to organs," Science, vol. 335, no. 6075, pp. 1458-1462, 2012.

[108] K. Pu, A. J. Shuhendler, J. V. Jokerst et al., "Semiconducting polymer nanoparticles as photoacoustic molecular imaging probes in living mice," Nature Nanotechnology, vol. 9, no. 3, pp. 233-239, 2014.

[109] D. Y. Lee, J. Y. Kim, Y. Lee et al., "Black pigment gallstone inspired platinum-chelated bilirubin nanoparticles for combined photoacoustic imaging and photothermal therapy of cancers," Angewandte Chemie International Edition, vol. 56, no. 44, pp. 13684-13688, 2017.

[110] H. S. Kim and Y.-H. Kim, "Recent progress in atomistic simulation of electrical current DNA sequencing," Biosensors and Bioelectronics, vol. 69, pp. 186-198, 2015.
[111] R. Drmanac, S. Drmanac, Z. Strezoska et al., "DNA sequence determination by hybridization: a strategy for efficient largescale sequencing," Science, vol. 260, no. 5114, pp. 1649-1652, 1993.

[112] M. Raoof, K. Jans, G. Bryce, S. Ebrahim, L. Lagae, and A. Witvrouw, "Improving the selectivity by using different blocking agents in DNA hybridization assays for SiGe biomolecular sensors," Microelectronic Engineering, vol. 111, pp. 421-424, 2013.

[113] J. Beheshtian, A. A. Peyghan, and M. Noei, "Sensing behavior of $\mathrm{Al}$ and $\mathrm{Si}$ doped $\mathrm{BC}_{3}$ graphenes to formaldehyde," Sensors and Actuators B: Chemical, vol. 181, pp. 829-834, 2013.

[114] A. Petranovska, N. V. Abramov, S. P. Turanska, P. P. Gorbyk, A. N. Kaminskiy, and N. V. Kusyak, "Adsorption of cisdichlorodiammineplatinum by nanostructures based on single-domain magnetite," Journal of Nanostructure in Chemistry, vol. 5, no. 3, pp. 275-285, 2015.

[115] A. Ahmadi, J. Beheshtian, and M. Kamfiroozi, "Benchmarking of ONIOM method for the study of NH 3 dissociation at open ends of BNNTs," Journal of Molecular Modeling, vol. 18, no. 5, pp. 1729-1734, 2012.

[116] J. Beheshtian, A. A. Peyghan, and Z. Bagheri, "A firstprinciples study of $\mathrm{H}_{2} \mathrm{~S}$ adsorption and dissociation on the AlN nanotube," Physica E: Low-dimensional Systems and Nanostructures, vol. 44, no. 9, pp. 1963-1968, 2012.

[117] L. Mahdavian, "Simulation of $\mathrm{SnO}_{2} / \mathrm{WO}_{3}$ nanofilms for alcohol of gas sensor based on metal dioxides: $\mathrm{MC}$ and LD studies," Journal of Nanostructure in Chemistry, vol. 3, no. 1, p. 1, 2012.

[118] A. A. Peyghan and M. Noei, "Fluorination of $\mathrm{BC}_{3}$ nanotubes: DFT studies," Journal of Molecular Modeling, vol. 19, no. 9, pp. 3941-3946, 2013.

[119] A. Das, A. K. Sood, P. K. Maiti, M. Das, R. Varadarajan, and C. N. R. Rao, "Binding of nucleobases with single-walled carbon nanotubes: theory and experiment," Chemical Physics Letters, vol. 453, no. 4-6, pp. 266-273, 2008.

[120] A. Rastgou, H. Soleymanabadi, and A. Bodaghi, "DNA sequencing by borophene nanosheet via an electronic response: a theoretical study," Microelectronic Engineering, vol. 169, pp. 9-15, 2017. 